This item was submitted to Loughborough's Research Repository by the author.

Items in Figshare are protected by copyright, with all rights reserved, unless otherwise indicated.

\title{
Landing site reachability in a forced landing of unmanned aircraft in wind
}

PLEASE CITE THE PUBLISHED VERSION

http://dx.doi.org/10.2514/1.C033856

\section{PUBLISHER}

(c) American Institute of Aeronautics and Astronautics

\section{VERSION}

AM (Accepted Manuscript)

\section{PUBLISHER STATEMENT}

This work is made available according to the conditions of the Creative Commons Attribution-NonCommercialNoDerivatives 4.0 International (CC BY-NC-ND 4.0) licence. Full details of this licence are available at: https://creativecommons.org/licenses/by-nc-nd/4.0/

\section{LICENCE}

CC BY-NC-ND 4.0

\section{REPOSITORY RECORD}

Coombes, Matthew, Wen-Hua Chen, and Peter M. Render. 2016. "Landing Site Reachability in a Forced Landing of Unmanned Aircraft in Wind". figshare. https://hdl.handle.net/2134/22827. 


\title{
Landing Site Reachability in a Forced Landing of
}

\section{Unmanned Aircraft in Wind}

\author{
Matthew Coombes ${ }^{1}$, Wen-Hua Chen $^{2}$, Peter Render ${ }^{3}$ \\ Department of Automotive and Aeronautical Engineering \\ Loughborough University, \\ Loughborough, LE11 3TQ UK
}

\begin{abstract}
Autonomous contingency management systems, such as a forced landing system which reacts appropriately to an engine failure is important for the safe operation of Unmanned Aircraft Systems (UAS). This paper details a method to ascertain the reachability of any possible emergency landing site for a forced landing in steady uniform wind conditions. With knowledge of the aircraft's state, such as speed heading location and orientation of a landing site, a method to calculate a minimum height loss path is developed based on aircraft glide performance. Wind direction and speed are taken into account using a trochoidal approach by defining the minimum height loss turn path. To facilitate real-time implementation, simplified gliding equations are developed without accuracy loss. The reachability of each site can be calculated, as well as how much safety margin an aircraft would have. This method is generic and could also provide decision support for human pilots in forced landing situations. Two types of aircraft Airbus A320-400 and the Cessna 172 have been investigated to demonstrate the usefulness of the method, using Monte Carlo simulations in a synthetic X-Plane ${ }^{\circledR}$ simulation environment, in order to demonstrate the performance and effectiveness of the proposed approaches.
\end{abstract}

\section{Index Terms}

Contingency management; Autonomous safety function; Unmanned aircraft systems; Reachability Analysis; Forced landing; Trochoids; X-Plane ${ }^{\circledR}$.

\section{INTRODUCTION}

The operation of Unmanned Aerial Systems (UASs) by the military has increased over the last couple of decades, driven by the advantages offered over the operation of manned aircraft. UAS not only removes the operator of the aircraft from

Research Associate, Dept. of Automotive and Aeronautical Engineering: ttmjc2@lboro.ac.uk ${ }^{1}$

Professor in Autonomous Vehicles, Dept. of Automotive and Aeronautical Engineering: w.chen@lboro.ac.uk ${ }^{2}$

Senior Lecturer in Aircraft Aerodynamics, Dept.of Automotive and Aeronautical Engineering: p.r.render@lboro.ac.uk ${ }^{3}$ 
danger but also, with the absence of pilots they have much longer endurance and a larger payload capacity. Consequently this makes them highly attractive for long surveillance or strike missions in dangerous areas. Many of the reasons identified by the military are also driving interest in the use of UAS for civilian applications. This triggers significant interest in developing safe UAS operations in civil airspace.

By removing the pilot, a number of safety issues are introduced. Among the most challenging is how a UAS would respond to engine failure so as to minimise the threat imposed on the public. As UASs tends to primarily be single engine powered aircraft, this makes them vulnerable to engine failures as any single engine powered General Aviation (GA) aircraft. Engine failure was the largest cause of GA accidents and the second largest killer of pilots in 2010 in the US. Out of 999 accidents involving GA aircraft, 180 were caused by engine failure [1]. This is why the forced landing situation is a major stumbling block to UAS integration into National Airspace System (NAS), and why a contingency management system is important in the event of engine failure [2]. It is imperative to develop autonomous functions to mitigate the risk imposed by UAV to the public in this scenario while reducing the loss of the aircraft and its payload. Such a system could also be used as a tool for assisting pilots in forced landing situations. An engine failure is a high work load situation, where the pilot may not have enough spare capacity to find a reachable and suitable emergency landing site. If a system could display all reachable and suitable sites, this could lead to pilots making better landing choices and consequently help save lives.

Upon an engine failure, an aircraft must carry out what is known as a forced landing. The aircraft, now unpowered, is effectively a glider and will need to make an emergency landing at an unprepared location. These landing sites are normally fields, many of which may be unsuitable due to size or obstacles. For a piloted aircraft, a pilot has to perform the forced landing by following a certain complex procedure which requires a great deal of practice for a human pilot to master. Considerable information is needed and a number of very complex decisions must be made in order to perform a successful forced landing within a very short time. Firstly, an engine failure needs to be identified. Then a list of possible landing sites is required, which can come from a number of sources, such as Geographic Information System (GIS), map data, pre-surveys, or live computer vision techniques. Computer vision techniques for selecting landing sites have been studied in [3], [4] and [5]. The list needs to be narrowed down to sites that are within the range of the aircraft, which requires an assessment of the reachability of each possible sites. Using data pertaining to this trimmed list of landing sites, a decision needs to be made at which of these sites to aim to land. A path then needs to be planned to the chosen landing site from the aircraft's current position that will get the aircraft lined up with the site at the correct altitude while accounting for wind and other uncertainties. Once the aircraft is on approach to the chosen site, a landing has to be performed.

The reachability of landing sites has been an area neglected in previous research, where a number of path planning techniques for forced landings assume that sites are within range [6], [7]. This is of paramount importance as there is very little point at aiming to land at the most perfect landing site if an aircraft is unable to reach it under the current aircraft status such as altitude, velocity and heading. Therefore it is essential to be able to calculate each known landing site's 
reachability. It is also important to show how reachable a landing site is, which is why the notion of excess glide range will be introduced to provide a measure safety margin for the forced landing, as well as describing how easily reachable a site is for use by a decision maker. To calculate this, the aircraft's glide performance, the minimum height loss path to fly, and path of the approach are all needed. References [8] and [9] lay out a number of equations for working out an aircraft's glide performance, as well as explaining the manoeuvres and speeds to minimise a gliding aircraft's height loss. The work in [9] aims to find at what point an aircraft after engine failure could not perform a turn back manoeuvre to land on the runway it took off from, this is extended in [10] and [11].

In some of the early work reported in [12], multiple sections of a forced landing system are examined for commercial aircraft typically cruising at high altitudes and with airports as the emergency landing sites. In what is called 'footprint generation', the maximum glide range of a gliding aircraft is defined based on simple glide angles. From a national database of runway locations, any runway that is within the footprint is considered reachable, as described in Fig. 1. While this will give an indication of a site's reachability, it does not include any kind of final manoeuvre to land at the site. For an aircraft with plenty of altitude and a small turn radius, this kind of method may be adequate as the error would be a small percentage of the maximum glide distance. However, this error would be unacceptable for a low-flying aircraft. For an unprepared landing site, visual inspection may be necessary. A method is presented in [8] to calculate the reachability of an unprepared landing site. It uses a human pilot forced landing approach technique called the 'high-key low-key' technique [13]. Combining with maximum glide range equations, it calculates the excess glide range of the aircraft for a selected landing site. The high-key low-key technique is intended to give a human pilot good visibility to study the site, and time to prepare the aircraft for landing. This may result in an inefficient use of the available glide range. A more generic technique for reachability analysis for autonomous forced landing is required.

Dubins Paths are the shortest curve that connects two points in the two-dimensional Euclidean plane [14]. This principle in this context means that the shortest path flown between a start and an end point must contain two turns at it's maximum turn rate, connected by a straight glide path between them, which makes a perfectly smooth trajectory. Therefore, upon engine failure, the aircraft turns onto a track which after a level glide intercepts the turn to final (final landing decent path to runway) at $500 \mathrm{ft}$. The final altitude of $500 \mathrm{ft}$ is chosen as the turn to the final should not be below $500 \mathrm{ft}$ for a normal landing [15]. This method based on Dubins can be used for any aircraft or runway orientation and for any radius of turn. This minimises the path length for an aircraft of any performance.

However, these circular flight paths do not take into consideration the effects of wind, which is very important for a gliding aircraft. The concept of Dubins curves is extended to take into account wind; instead of the straight glide line being tangential to two circles, it is tangential to two geometric shapes called trochoids. Trochoids are the curve described by a fixed point on a circle as it rolls along a straight line, which are defined later. Trochoids are used for path planning in the presence of wind in [16] and [17], where minimum flight time path planning is considered. This paper will extend these 


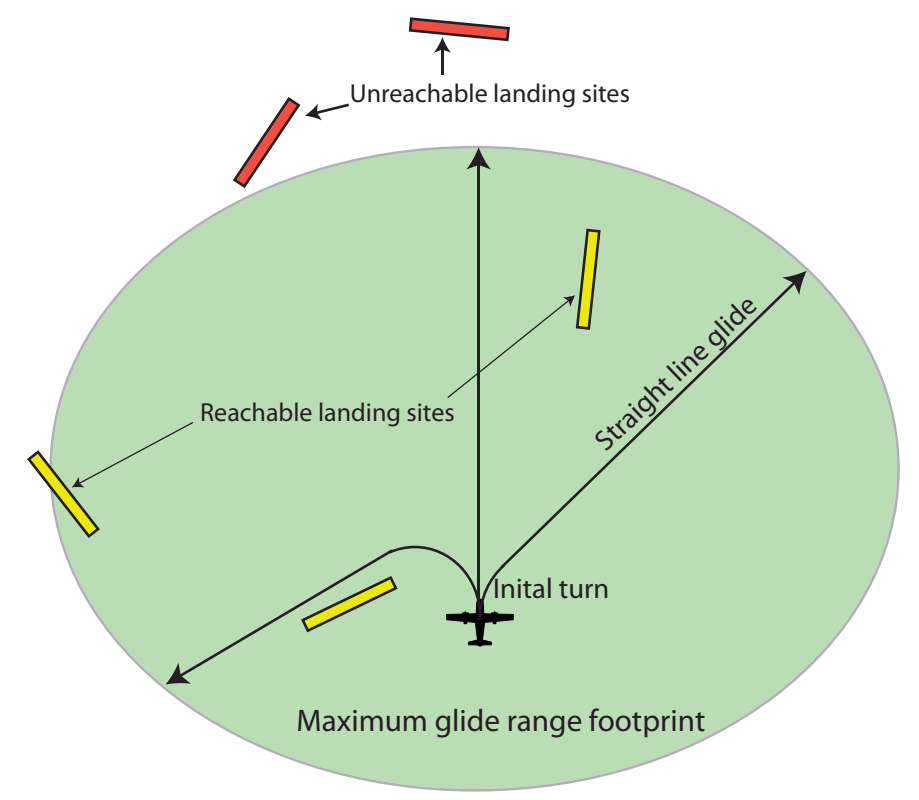

Fig. 1: Maximum glide range footprint and how previous work has assessed landing site reachability in a forced landing.

to define the minimum height loss flight path in wind by determining the speeds and turn radius for a given aircraft, and analyse reachability of a site for forced landing. An example of this extension of Dubins paths to trochoidal paths is shown in Fig. 2.

The contribution to this research area is to extend the simplistic concept of glide range footprint to include the approach to the site and wind. Neither of these have ever been induced in previous glide range calculation algorithms. Importantly this technique also includes a metric for comparing the reachability of landing sites. This is in order to show how much safety margin a gliding aircraft has, as well as to provide this information to other parts of a full forced landing system, e.g. landing site decision maker.

In Section II the equations describing the glide performance of an aircraft in both straight and level, and turning flight are defined. Presented in Section III are a set of equations to define the minimum height loss path in the presence of steady wind. Section IV shows how the trochoidal path can be used with the glide performance equations to ascertain if a particular landing site is reachable and how much excess glide distance it has when it reaches it. To verify the proposed method, two different types of aircraft are considered: Airbus 320-400 and Cessna 172. In Section V the accident of the Hudson River is investigated, whereby where the reachability of the runway it took off from is assessed and the influence of the wind on reachability is highlighted. The proposed method is further demonstrated and evaluated in Section VI where simulation and analysis are carried out in a high fidelity X-Plane ${ }^{\circledR}$ simulation environment. After determining the gliding performance experimentally, the accuracy and real-time property have been investigated. The influence of the assumptions and simplifications such as instantaneous rolling in and out used in developing the algorithm are assessed. Finally concluding remarks are presented in Section VII. 


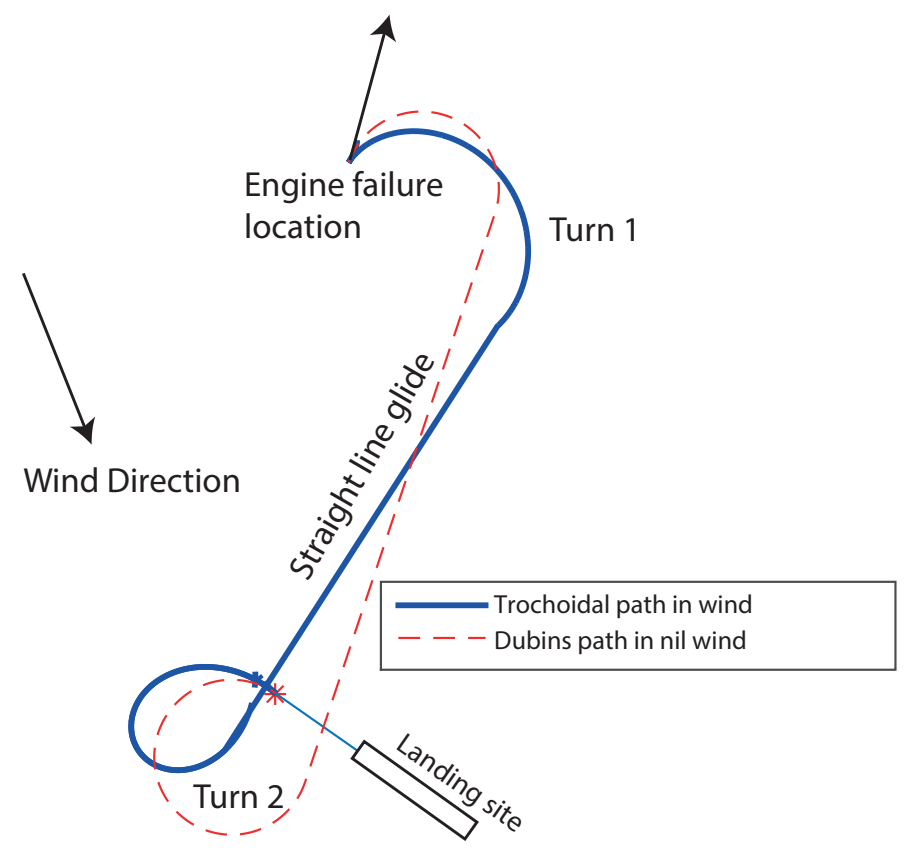

Fig. 2: Comparison between a left straight left Dubins path in nil wind to a trochoidal path in wind in a forced landing scenario.

\section{GLIDE PERFORMANCE}

Before any glide paths or approach paths are defined, the glide performance of the aircraft needs to be known. The vertical sink $\left(V_{s}\right)$ for a given airspeed is needed. The aircraft's lift/drag ratio is the glide ratio of aircraft, and a number of factors will influence both of these, the main being the aircraft's drag polar. The optimum speeds and roll angles for the turn manoeuvres are needed to maximise glide range so that the minimum length path is also the minimum height loss path.

Calculations are put forward in [8] to calculate the glide ratio and vertical sink of a gliding aircraft. The calculation for $V_{s}$ presented assumes that the aircraft remains at best glide speed throughout the flight. The best glide speed is the airspeed that the aircraft needs to fly at which maximises its glide ratio. However, in a co-ordinated turn, this speed increases due to the higher levels of lift and therefore increases drag from the greater normal loading $(n)$. It is assumed that the aircraft would speed up in turns to maintain best glide for these new conditions. This is not what an aircraft would actually do and the airspeed transition would add inaccuracies into the equations, so a modified equation is shown here that gives the vertical sink of the aircraft for any airspeed, not simply the optimum. There may exist some airspeed profile that would maximise the aircraft glide range further through the transition from turning to straight and level flight. However, only a constant airspeed is considered to significantly reduce complexity, and so maintain the possibility of real time calculations.

To maintain airspeed without thrust the aircraft must remain in equilibrium by cancelling the drag force with a component from its weight. By assuming a small angle approximation on the glide path the vertical speed can be calculated by: 


$$
V_{s}=\frac{D V}{W}
$$

where $D$ is drag, $V$ is airspeed and $W$ is the weight of the aircraft.

Using Eq. (1) with an aircraft's parabolic drag polar, $V_{s}$ can be calculated in straight and level flight as shown in [8] the vertical speed can be calculated by:

$$
V_{s}=A V^{3}+\frac{B}{V}
$$

with

$$
A=\frac{0.5 \rho_{0} S C_{D o}}{W}
$$

and

$$
B=\frac{2 W}{\rho_{0} S \pi A_{r} e}
$$

$A_{r}$ is the aspect ratio, $\rho_{0}$ is the density of air at sea level, $C_{D o}$ is the drag at zero lift, $e$ is Oswald Efficiency Factor, and $S$ is the wing area. It is assumed aircraft instantaneously attains the desired roll angle.

The maximum glide range speed $\left(V_{i o}\right)$ can be found by differentiating $\frac{V}{V_{s}}$ which is the definition of glide ratio $(\gamma)$, where 2 can be rearranged to give:

$$
\gamma=\frac{V}{V_{s}}=\frac{1}{A V^{2}}+\frac{V^{2}}{B}
$$

to

$$
\frac{d \gamma}{d V}=-\frac{2}{A V^{3}}+\frac{2 V}{B}=0
$$

To give the optimum airspeed from aircraft parameters below:

$$
V_{i o}=\left(\frac{B}{A}\right)^{\frac{1}{4}}
$$

For a co-ordinated turning manoeuvre, the sink increases as $n$ increases from unity, meaning that $L=n W$. This can be substituted in to Eq. (1) to give Eq. (8).

$$
V_{s \phi}=\frac{D V n}{L}
$$

where $V_{s \phi}$ is the vertical sink in a turn.

Normal loading in a turn is equivalent to $\sec (\phi)$ where $\phi$ is the roll angle. Substituting $C_{L}$ for lift and the parabolic drag polar $C_{D}=C_{D o}+\frac{C_{L}^{2}}{\pi e A_{r}}$ in to Eq. (8), the full equation for sink in a turn for any airspeed or roll angle is given by

$$
V_{s \phi}=A V^{3}+\frac{B \sec ^{2}(\phi)}{V}
$$




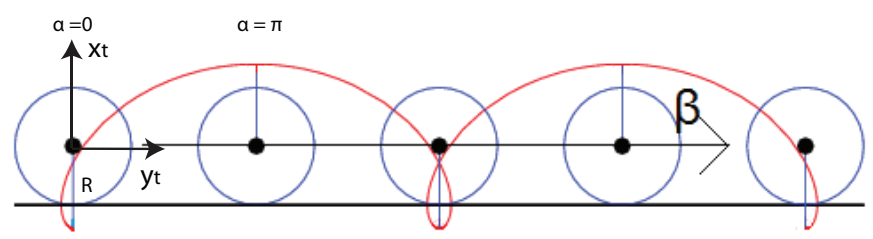

Fig. 3: A circle moving at a fixed rate $\beta$, while tracing a trochoid at a point at a fixed radius.

\section{TROCHOIDAL TURN PATH}

A turn at a constant velocity and roll angle in no wind (assuming instantaneous roll) will be a perfect circle. An initial turn circle and a final turn circle linked by a tangent would define the minimum length path in no wind. In the presence of wind, the aircraft is no longer in the earth frame, but in the wind frame. This means the circle becomes a shape known as a trochoid. Tangents can be calculated to link the first and second trochoidal turn path into a single smooth trajectory.

A trochoid is a curve traced by a point on a radius of a circle, where the centre of the circle is moving at a fixed rate $(\beta)$ along a straight line. This is shown in Fig. 3. The shape can be defined parametrically by angle $(\alpha)$ as the input in Eq. (10). $\alpha$ can be related to the heading of the aircraft, but as it is used slightly differently between the initial and final trochoids, how it can be used to ascertain the heading will be explained later.

$$
\begin{array}{r}
x_{t}=-R \cos (\alpha) \\
y_{t}=R \sin (\alpha)+R \beta \alpha
\end{array}
$$

The aircraft flies a circular path in air but as this is in the wind axes, the transformation to Earth axes means that the aircraft is in fact flying a trochoidal path. This is because the turn circle centre is being blown down wind at the rate $\beta$ which is the ratio of windspeed to the aircraft's airspeed, shown in Eq. (11).

$$
\beta=\frac{V_{w}}{V_{i o}}
$$

where $V_{w}$ is the wind velocity.

Eq. (10) is the parametric equation for a turn circle with radius $R$, the $R \beta \alpha$ term represents the aircraft getting blown downwind ( $Y_{t}$ axis) during its turn.

The trochoidal path method described in [16] has been adapted for use here. However the aim of their paper is to seek a time optimal path for a given set of initial and final conditions. Angles are more useful in the forced landing application, as it can be directly related to the track angle of the aircraft. All equations are re-derived to use angle. 


\section{A. Trochoid definitions and positions}

The trochoid is defined in the trochoidal frame, which is where the $y$ direction is aligned with the wind. However the trajectories in the trochoidal frame will have to be rotated to put them in the earth frame.

The path for the whole flight must be defined with only the initial, and final conditions of the aircraft. These include initial aircraft heading $\left(\psi_{0}\right)$, final aircraft heading, which is the runway landing direction $(\Gamma)$ and the position of the turn to final waypoint $\left(F_{x}, F_{y}\right)$. The turn to the final waypoint is calculated in Eq. (12), where the waypoint is defined relative to the centreline of the runway at a longitudinal distance that would enable the aircraft to glide to the runway's centre from a height of 500ft. It is then transformed into the global co-ordinates by rotating the runway direction.

$$
\left[\begin{array}{l}
F_{x} \\
F_{y}
\end{array}\right]=\left[\begin{array}{cc}
\cos \Gamma & -\sin \Gamma \\
\sin \Gamma & \cos \Gamma
\end{array}\right]\left[\begin{array}{c}
0 \\
0.3048 \times 500 \gamma
\end{array}\right]+\left[\begin{array}{l}
x_{r} \\
y_{r}
\end{array}\right]
$$

where $0.3048 \times 500 \gamma$ is the distance an aircraft will travel from a height of $500 \mathrm{ft}$ at it's glide ratio $(\gamma)$.

As $\left(F_{x}, F_{y}\right)$ are in the Earth frame and need to be rotated into the trochodial frame, they must be rotated by the wind direction $\left(\psi_{w}\right)$ shown by:

$$
\left[\begin{array}{l}
F_{t x} \\
F_{t y}
\end{array}\right]=\left[\begin{array}{cc}
\cos \psi_{w} & -\sin \psi_{w} \\
\sin \psi_{w} & \cos \psi_{w}
\end{array}\right]\left[\begin{array}{l}
F_{x} \\
F_{y}
\end{array}\right]
$$

There will be two trochoids; one for the initial turn and one for the final turn. The equations for these need to be defined using the initial and final conditions. From considering aircraft turn performance, the radius of the turn used in Eq. (10) is defined as:

$$
R=\frac{V_{i o}}{\dot{\psi}}
$$

where $\dot{\psi}$ is the yaw rate of the aircraft, and the aircraft is flying at maximum glide speed $V_{i o}$.

The angle $\alpha$ needs to be adjusted by the phase angle $\eta$ to account for the transformation between frames, and for the initial and final headings

$$
\eta_{1}=\psi_{0}-\psi_{w}, \quad \eta_{2}=\Gamma-\psi_{w}-\delta_{2} 2 \pi
$$

$\eta_{1}$ is the phase angle for the initial turn and $\eta_{2}$ for the final turn and $\delta$ is the sign of the turn direction. The term $\delta_{2} 2 \pi$ is added to the second turn as the aircraft needs to be at $\Gamma$ at the end of the trochoid, which is at $2 \pi$.

The start of the first trochoid must be placed at the point where the aircraft starts $\left(x_{0}, y_{0}\right)$. It is easier however to have the aircraft start at $(0,0)$ which will be used here. Similarly the end of the second trochoid must be located at $\left(F_{t x}, F_{t y}\right)$. They are repositioned by calculating the co-ordinates of the start of the initial trochoid and the end of the final trochoid, the positioning of these trochoids can be shown in Fig. 4. 


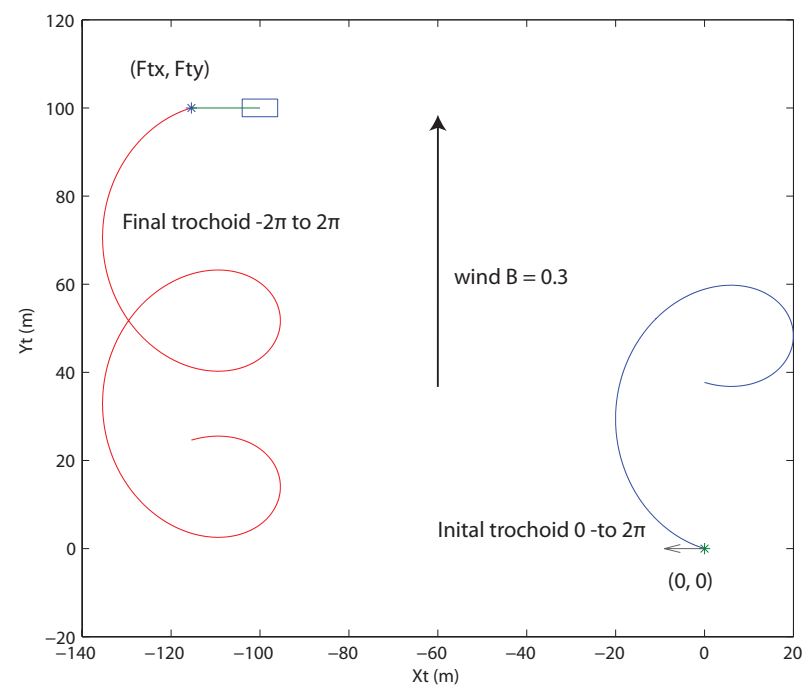

(a) Trochoidal frame.

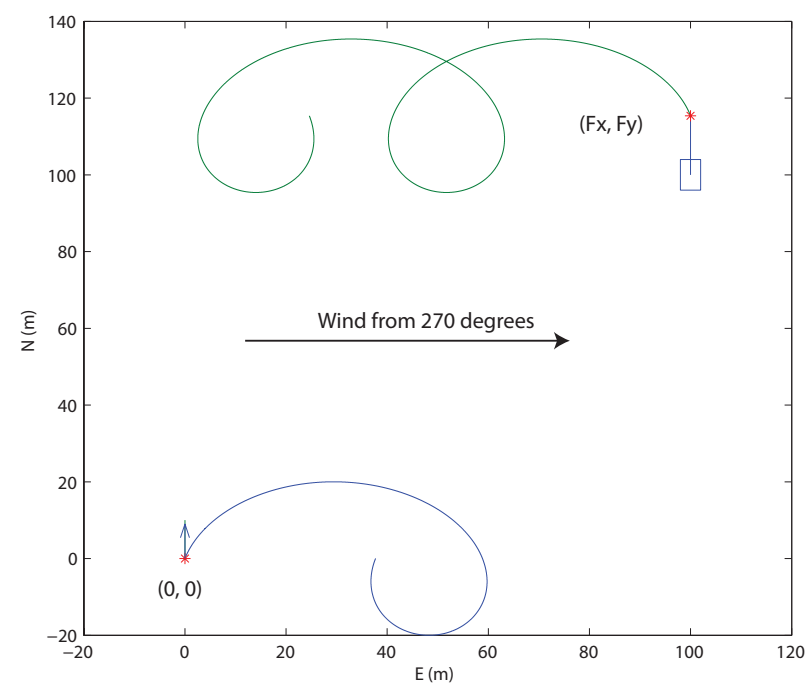

(b) Earth frame.

Fig. 4: Shows the position of both trochoids in the trochoidal and earth frame. As the initial conditions have been rotated to the trochoidal frame, therefore once the paths are defined, they can simply be rotated about the starting point of the aircraft.

The initial and final trochoid can be calculated parametrically in the trochoidal frame by substituting Eq. (14), Eq. (15), into Eq. (10). This gives the Cartesian co-ordinates for the initial trochoid for a given $\alpha$ in:

$$
\begin{array}{r}
x_{t 1}=-\frac{V_{i o}}{\delta_{1} \dot{\psi}} \cos \left(\alpha+\eta_{1}\right)+x_{t 0} \\
y_{t 1}=\frac{V_{i o}}{\delta_{1} \dot{\psi}} \sin \left(\alpha+\eta_{1}\right)+\frac{V_{i o}}{\delta_{1} \dot{\psi}} \beta \alpha+y_{t 0}
\end{array}
$$

and for the final trochoid in:

$$
\begin{array}{r}
x_{t 2}=-\frac{V_{i o}}{\delta_{2} \dot{\psi}} \cos \left(\alpha+\eta_{2}\right)+x_{t f} \\
y_{t 2}=\frac{V_{i o}}{\delta_{2} \dot{\psi}} \sin \left(\alpha+\eta_{2}\right)+\frac{V_{i o}}{\delta_{2} \dot{\psi}} \beta \alpha+y_{t f}
\end{array}
$$

$\frac{V_{i o}}{\delta_{1} \dot{\psi}}$ is the radius of the turn adjusted for the turn direction $\delta$. Each of the two turns can be in either direction. These directions are represented by $\delta_{1}, \delta_{2}$ which are either -1 for an anti clockwise direction, and 1 for clockwise, where there are four possible trajectories. $\left(x_{t} 0, y_{t} 0\right)$ is the offset of the initial trochoid to place the start of the trochoid at the initial position of the aircraft, $\left(x_{t f}, y_{t f}\right)$ is the offset of the final trochoid to place the end of the trochoid at $\left(F_{t x}, F_{t y}\right)$. These are defined below:

$$
\begin{gathered}
x_{t 0}=\frac{V_{i o}}{\delta_{1} \dot{\psi}} \cos \left(\eta_{1}\right)+x_{0} \\
y_{t 0}=-\frac{V_{i o}}{\delta_{1} \dot{\psi}} \sin \left(\eta_{1}\right)+y_{0}
\end{gathered}
$$




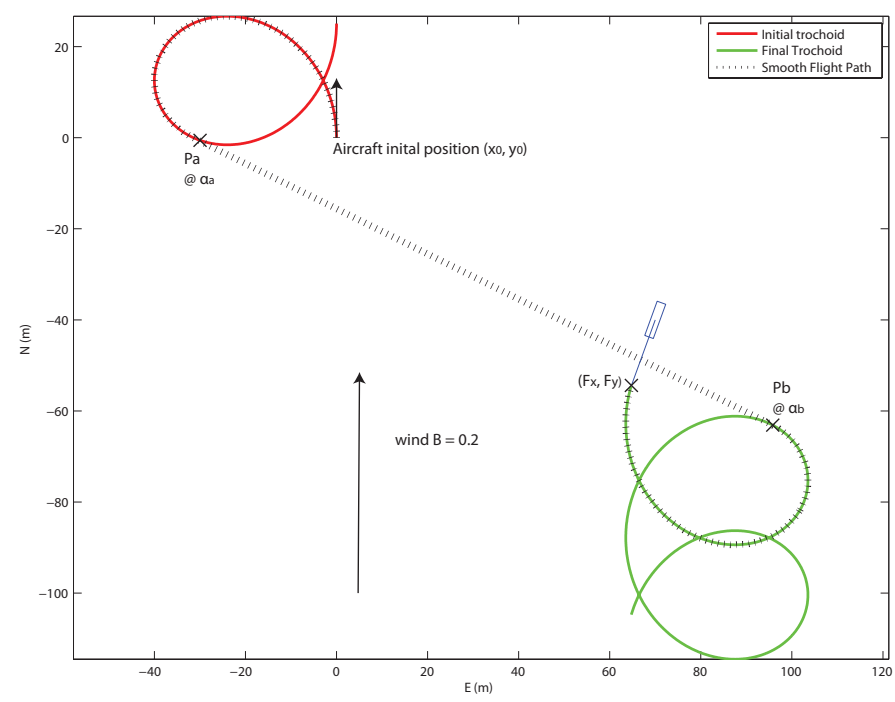

Fig. 5: Anti-clockwise/clockwise flight path, where both trochoids and the optimal tangent between them have been defined. The tangent starts at $P_{a}$ and finishes as $P_{b} . \psi_{0}=0 \Gamma=\frac{\pi}{6}$

$$
\begin{array}{r}
x_{t f}=\frac{V_{i o}}{\delta_{2} \dot{\psi}} \cos \left(\eta_{2}\right)+F_{t x} \\
y_{t f}=\frac{V_{i o}}{\delta_{2} \dot{\psi}} \sin \left(\eta_{2}\right)+2 \frac{V_{i o}}{\delta_{2} \dot{\psi}} \beta \pi+F_{t y}
\end{array}
$$

How the two trochoids are positioned, plotted, and then rotated is shown in Fig. 4. The initial and final trochoid are plotted relative to the aircraft's initial position and the final waypoint $\left(F_{t x}, F_{t y}\right)$ in the trochoidal frame. Everything is then rotated to be in the Earth frame. The initial and final conditions in Fig. 4 are shown below:

$$
\begin{array}{ll}
\psi_{0}=0 & \Gamma=\pi \quad F_{x}=100 \quad F_{y}=115.4 \quad x_{0}=0 \\
y_{0}=0 & \psi_{w}=\frac{\pi}{2}
\end{array}
$$

where the wind must be converted into the direction of travel, so in this case the wind is from $\frac{3 \pi}{2}$ so travels at $\frac{\pi}{2}$.

For an optimal path the total angle change over both turns must be less than $4 \pi$ which is proven in [16]. This is why the in Fig. 4 initial trochoid is plotted for $\alpha$ between $0 \rightarrow 2 \pi$, and the final trochoid is plotted between $-2 \pi \rightarrow 2 \pi$. For the trajectory to be optimal the tangent will lay somewhere between these two trochoids.

\section{B. Finding tangents between trochoids}

The two trochoids now need to be connected with a straight and level glide between them, which will create a smooth trajectory. The departure point from the initial trochoid will be referred to as $P_{a}$, which will occur at angle $\alpha_{a}$. Similarly, at the end of the straight glide, the join point on the final trochoid is referred to as $P_{b}$ at an angle of $\alpha_{b}$. It will be these two angles which must be found to define the whole path. This can be seen in Fig. 5. 


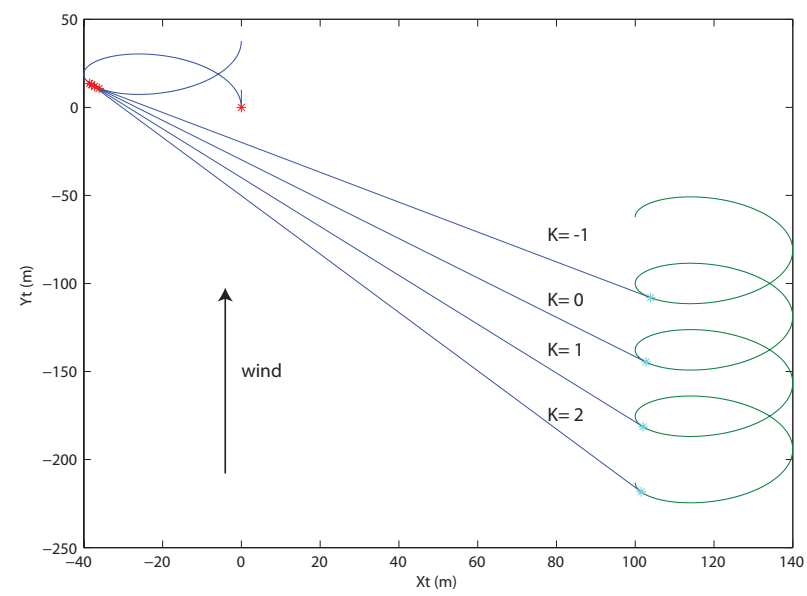

Fig. 6: The effect of $k$.

For a tangent to exist the aircraft's ground track $\left(\psi_{g}\right)$ at both point $P_{a}$ and $P_{b}$ must be the same, as well as their velocity vectors shown in Eq. (21) and (22). Both $\alpha_{a}$ and $\alpha_{b}$ need to be found so that the $(x, y)$ co-ordinates for $P_{a}$ and $P_{b}$ can be found from Eq. (18), and (19).

$$
\begin{gathered}
\tan \left(\psi_{g}\right)=\frac{y\left(\alpha_{b}\right)-y\left(\alpha_{a}\right)}{x\left(\alpha_{b}\right)-x\left(\alpha_{a}\right)} \\
\tan \left(\psi_{g}\right)=\frac{\dot{y}\left(\alpha_{b}\right)}{\dot{x}\left(\alpha_{b}\right)}=\frac{\dot{y}\left(\alpha_{a}\right)}{\dot{x}\left(\alpha_{a}\right)}
\end{gathered}
$$

By substituting Eq. (16) and (17), into Eq. (21), a full equations for the heading of the tangent can be found in Eq. (23).

$$
\tan \left(\psi_{g}\right)=\frac{\frac{V}{\delta_{2} \dot{\psi}} \sin \left(\alpha_{b}+\eta_{2}\right)+\frac{V}{\delta_{2} \dot{\psi}} \beta \alpha_{b}+y_{t 2}-\frac{V}{\delta_{1} \dot{\psi}} \sin \left(\alpha_{a}+\eta_{1}\right)+\frac{V}{\delta_{1} \dot{\psi}} \beta \alpha_{a}+y_{t 1}}{-\frac{V}{\delta_{2} \dot{\psi}} \cos \left(\alpha_{b}+\eta_{2}\right)+x_{t 2}+\frac{V}{\delta_{1} \dot{\psi}} \cos \left(\alpha_{a}+\eta_{1}\right)+x_{t 1}}
$$

The relationship between $\alpha_{a}$ and $\alpha_{b}$ needs to be found in order to simplify Eq. (23) to put it in terms of only $\alpha_{a}$. The time version of this equation is described in [16], and is converted to the angular version below

$$
\alpha_{b}=\frac{\delta_{1}}{\delta_{2}} \alpha_{a}+\frac{\eta_{1}-\eta_{2}+2 k \pi}{\delta_{2}}
$$

This is derived by knowing that the total angle travelled by the aircraft around both turns must be equal to $\psi_{0}-\Gamma$, as this is the minimum angle to get the aircraft from the initial heading to the final heading.

As a trochoid is based on a periodic function, there will be an infinite number of tangents between both trochoids. Only solutions within a certain range are needed to keep the path optimal, but there may be multiple solutions within this range, to find them, multiples of $2 k \pi$ will be added (seen in Eq. (24)) where $k \in[-3,-2,-1,0,1,2]$. The effect of $\mathrm{k}$ is shown in Fig. 6. Increasing $\mathrm{k}$ by 1 shifts the angle of tangency to the next loop on the final trochoid. 


$$
\tan \left(\psi_{g}\right)=\frac{\left(x_{t 2}-x_{t 1}\right)+V \frac{\delta_{2}-\delta_{1}}{\delta_{2} \delta_{1} \dot{\psi}} \cos \left(\delta_{1} \alpha_{a}+\eta_{1}\right)}{\frac{\delta_{2}-\delta_{1}}{\delta_{2} \delta_{1} \dot{\psi}} \sin \left(\delta_{1} \alpha_{a}+\eta_{1}\right)+\left(y_{t 2}-y_{t 1}\right)+\frac{V_{w}}{\dot{\psi}}\left(\alpha_{a}\left(\frac{\delta_{1}}{\delta_{2}}-1\right)+\frac{\eta_{1}-\eta_{2}+2 k \pi}{\delta_{2}}\right)}
$$

A full equation for the angle of tangency between the two trochoids is needed. The $\alpha_{b}$ terms from Eq. (23) can be substituted for $\alpha_{a}$ using Eq. (24), the result is shown in Eq. (25) (see next page).

Now by equating Eq. (25), with Eq. (23) which removes the $\tan \left(\psi_{g}\right)$ term, and by using the identity $\sin (\psi)^{2}+\cos (\psi)^{2} \equiv 1$ the whole equation can be simplified to Eq. (26).

$$
\begin{gathered}
V\left(V_{w} \frac{\delta_{1}-\delta_{2}}{\delta_{1} \delta_{2} \dot{\psi}}-\left(x_{t 2}-x_{t 1}\right)\right) \cos \left(\delta_{1} \alpha_{a}+\eta_{1}\right) \\
+V\left(\left(y_{t 2}-y_{t 1}\right)+\frac{V_{w}}{\dot{\psi}}\left(\alpha_{a}\left(\frac{\delta_{1}}{\delta_{2}}-1\right)+\frac{\eta_{1}-\eta_{2}+2 k \pi}{\delta_{2}}\right)\right) \\
\sin \left(\left(\delta_{1} \alpha_{a}+\eta_{1}\right)\right)-V_{w}\left(x_{t 2}-x_{t 1}\right)-\frac{V^{2}\left(\delta_{2}-\delta_{1}\right)}{\delta_{2} \delta_{1} \dot{\psi}}=0
\end{gathered}
$$

There is no analytical solution for $\alpha_{a}$, as this equation is transcendental. However, in the next two sections, solving this equation and finding the points of tangency between the two trochoids will be shown.

\section{Solving for same side turns}

As both turns can be in either directions, there are four different combinations. When the turns are on the same side, i.e. clockwise/clockwise or anti-clockwise/anti-clockwise, this means $\delta_{1}=\delta_{2}$. Consequently, Eq. (25) can be simplified to Eq. (27). The extra term $2 \pi m$ has been added. It works similarly to k but instead of shifting the point $P_{b}$ to the next trochoidal loop on the second trochoid, it shifts both $P_{a}$ and $P_{b}$ to the next loop on both trochoids. As there are infinite solutions, both $\mathrm{k}$ and $\mathrm{m}$ must be changed in order to find to optimum path. As $\psi_{g}=\alpha_{a}, \alpha_{a}$ can be found.

$$
\tan \left(\alpha_{a}\right)=\frac{\left(x_{t 1}-x_{t 1}\right)}{\left(y_{t 2}-y_{t 1}\right)+V\left(\frac{\eta_{1}-\eta_{2}+2 k \pi}{\delta_{2}}\right)}+2 \pi m
$$

Eq. (27) can be easily solved for $\alpha_{a}$, and by using Eq. (24), $\alpha_{b}$ can be found. A range of values for $\mathrm{k}$ and $\mathrm{m}$ need to be used to find the path that satisfies the conditions in Eq. (28). This will make the path optimal.

$$
\left\{\begin{array}{c}
0<\alpha_{a}<2 \pi \\
-2 \pi<\alpha_{b}<2 \pi
\end{array}\right.
$$

\section{Solving numerically for different side turns}

As $\delta_{1} \neq \delta_{2}$ Eq. (26) must be used to find the tangent angle $\alpha_{a}$ from the first trochoid to the second. The roots $\alpha_{a}$ in the equation need to be found between the range $0 \rightarrow 2 \pi$ to make the trajectory optimum, as is proved in [16]. The roots can be found when $f\left(\alpha_{a}\right)=0$, and any root finding technique can be used. Here the bisection method is used. A good 
introduction to the bisection method is contained in [18]. The bisection method finds all the values of $x$ where $f(x)=0$ between an interval $[a, b]$. If $f(a)$ and $f(b)$ have opposite signs, this means that they are between a root, and it is on this principle that the algorithm works.

As mentioned previously, a range of $k[-1,0,1,2,3]$ values need to be used in order to find the best tangent to make the flight path as short as possible.

In the interval $[a, b]$ (for a given value of $k$ ) there may be multiple roots, but some of these will create sub optimal paths, or infeasible flight paths. Now that a range of exit angles are known for the first trochoid $\left(\alpha_{a}\right)$, these will be narrowed down to the shortest legitimate flight path. To eliminate the invalid paths, the angle of the tangent from $P_{a}$ to $P_{b}$ will be compared to the track of the aircraft at point $P_{a}$. To find the aircraft's track, the aircraft's $x$ and $y$ velocities can be used with the 4-quadrant inverse tangent function (atan2). The aircraft velocities are obtained from differentiating Eq. (16) to give Eq. (29).

$$
\begin{array}{r}
V_{t x a}=V_{i o} \sin \left(\alpha_{a}+\eta_{1}\right) \\
V_{t y a}=V_{i o} \cos \left(\alpha_{a}+\eta_{1}\right)+V_{w}
\end{array}
$$

where $V_{t x a}$ and $V_{t y a}$ are the aircraft $x$, and $y$ velocities in the trochodial frame at point $P_{a}$. All these checks will be performed in the trochoidal frame for simplicity. The aircraft is flown at the maximum glide range speed of $V_{i o}$ around the whole flight path, which is why it is used here.

The track of the aircraft can be calculated by:

$$
\begin{gathered}
\psi_{t g}=\arctan \left(\frac{V_{x t a}}{V_{y t a}}\right)=\arctan \left(\frac{V_{i o} \sin \left(\alpha_{a}+\eta_{1}\right)}{V_{i o} \cos \left(\alpha_{a}+\eta_{1}\right)+V_{w}}\right) \\
\theta_{t a n g}=\operatorname{atan}\left(\frac{x_{t P b}-x_{t P a}}{y_{t P b}-y_{t P a}}\right)
\end{gathered}
$$

where $\left(x_{t P a}, y_{t P a}\right)$ is the co-ordinate of $P_{a}$ at the point tangent meets the first trochcoid, and $\left(x_{t P a}, y_{t P b}\right)$ the co-ordinates at point $P_{b}$. $\theta_{\text {tang }}$ is the angle of the tangent in the trochoidal frame from $P_{a}$ to $P_{b}$.

If $\psi_{g}=\theta_{\text {tang }}$, then that particular flight path is valid. As can be seen in Fig. 7 , the invalid path has a $\psi_{g}=91^{\circ}$, but the tangent is $180^{\circ}$ off at $\theta_{\text {tang }}=271^{\circ}$. The shortest path out of the valid ones has the largest $\alpha_{b}$ angle. This is because the closer to $2 \pi \alpha_{b}$ is, the smaller the angle the aircraft will have to subtend around the second trochoid. This can be clearly seen in Fig. 7. The two valid paths show their $\alpha_{b}$ values and the path labelled as the shortest has a much higher $\alpha_{b}$ value than the other. As the angle $\alpha_{a}$ is constrained to between $0 \rightarrow 2 \pi$ this has been already been optimised for a given $\alpha_{b}$. 


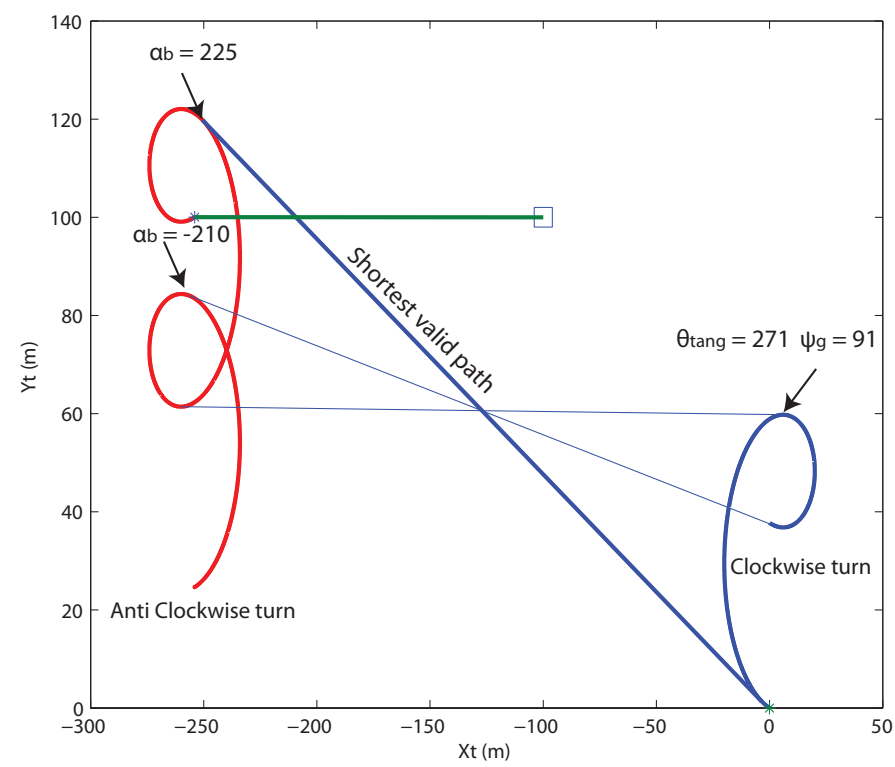

Fig. 7: There are 3 tangents between both trochoids for $\mathrm{k}=0$, one path is not a legitimate flight path, one is sub-optimal and the other is tangent chosen as the flight path, as it give the shortest distance.

\section{EXCESS GLIDE, AND ARC LENGTH CALCULATIONS}

After the path has been defined, the distance travelled, the height lost, and excess glide range now need to be calculated. To find the distance travelled, the arc length (L) needs to be calculated for both turns, as well as the straight glide. As the turn is now defined by a trochoid, a new equation is needed for arc length. For parametric equations, arc length is defined below by:

$$
L=\int \sqrt{\frac{d x^{2}}{d \psi}+\frac{d y^{2}}{d \psi}} d \psi
$$

Differentiating Eq. (10) with respect to $\psi$ gives:

$$
\begin{array}{r}
\frac{d x}{d \psi}=\delta R \cos (\psi) \\
\frac{d y}{d \psi}=\delta R \sin (\psi)+\delta R \beta
\end{array}
$$

Substituting Eq. (33) in to Eq. (32) yields Eq. (34).

$$
\sqrt{\frac{d x^{2}}{d \psi}+\frac{d y^{2}}{d \psi}}=R \sqrt{\sin (\psi)^{2}+\cos (\psi)^{2}+2 \beta \cos (\psi)+\beta^{2}}
$$

which can be simplified to give the full integral for arc length shown below:

$$
L=\int_{0}^{\psi} R \sqrt{1+2 \beta \cos (\psi)+\beta^{2}}
$$


Eq. (35) cannot be solved analytically but as these are a common type of integration, a set of functions exist to deal with them. The functions are called elliptic integrals and are laid out in [19]. The particular function is an incomplete elliptic integral of the second kind which is defined in Eq. (36).

$$
E(\psi \mid m)=\int_{0}^{\psi} \sqrt{1-m \sin (\psi)^{2}} d \psi
$$

Although this is not in the exact same form as Eq. (35), by rearranging the input into this function, one can show

$$
\int_{0}^{\psi} R \sqrt{1+2 \beta \cos (\psi)+\beta^{2}}=2 R(1+\beta) E\left(\frac{\psi}{2} \mid \frac{4 \beta}{(1+\beta)^{2}}\right)
$$

Shown in Eq. (38) and (39) are the full definite integrals with the correct limits for the arc lengths for both trochoids.

$$
L_{a}=2 R(1+\beta) E\left(\frac{\alpha_{a}}{2} \mid \frac{4 \beta}{(1+\beta)^{2}}\right)-2 R(1+\beta) E\left(0 \mid \frac{4 \beta}{(1+\beta)^{2}}\right)
$$

where $L_{a}$ is the arc length subtended by the aircraft around the first trochoid.

$$
L_{b}=R(1+\beta) E\left(2 \pi \mid \frac{4 \beta}{(1+\beta)^{2}}\right)-2 R(1+\beta) E\left(\frac{\alpha_{b}}{2} \mid \frac{4 \beta}{(1+\beta)^{2}}\right)
$$

where $L_{b}$ is the arc length subtended by the aircraft around the second trochoid.

The total ground distance covered by the aircraft is shown in Eq. (40).

$$
L=L_{a}+L_{b}+\sqrt{\left(y_{t P b}-y_{t P a}\right)^{2}+\left(x_{t P b}-x_{t P a}\right)^{2}}
$$

Height loss around the turn needs to be calculated so the landing site's reachability and the excess glide range can be ascertained. Knowing the glide ratio and glide distances, height loss across a glide can be calculated in still wind. When factoring in wind, the aircraft's ground speed is not constant around the turn, which means glide ratio is also not constant. As the vertical sink of the aircraft is calculated in the wind frame the height loss in the turn can be found from time in the turn.

As it is assumed that the aircraft can instantaneously achieve a given roll angle, This means that the time taken in the turn phases can be calculated by using the aircraft's yaw rate $\dot{\psi}$. This calculation is shown in Eq. (41) where $\frac{V}{R}=\dot{\psi}$. While ground speed is not constant, the aircraft sink will be constant as a steady airspeed is being maintained.

$$
t_{a}=\frac{V}{R} \alpha_{a}
$$

where $t_{a}$ is the time the aircraft takes to travel around the first turn.

The time taken around the second turn $\left(t_{b}\right)$ is dealt with in Eq. (42), which is a modified version of Eq. (24). 


$$
t_{b}=\frac{2 \pi}{\dot{\psi}}-t_{a}-\frac{\eta_{1}-\eta_{2}+2 k \pi}{\delta_{2} \dot{\psi}}
$$

$t_{a}$ and $t_{b}$ can be used alongside Eq. (9) for aircraft sink to calculate height lost in the turn shown below:

$$
\Delta h_{a}=V_{s \phi} t_{a}
$$

where $V_{s \phi}$ is the aircraft's vertical sink in a turn, $\Delta h_{a}$ the height lost around the first turn, and $t_{a}$ can simply be replaced with $t_{b}$ to get the height lost around the second turn.

The height loss in the straight and level glide between both turns $\left(\Delta h_{g}\right)$ is calculated below:

$$
\Delta h_{g}=V_{s} \frac{\sqrt{\left(x_{t P b}-x_{t P a}\right)^{2}+\left(y_{t P b}-y_{t P a}\right)^{2}}}{\sqrt{V_{t y a}^{2}+V_{t x a}^{2}}}
$$

where the numerator represents the distance travelled over ground, the denominator is the aircraft's ground speed and $V_{s}$ is the sink calculated from Eq. (2).

The total loss of height over the entire trajectory $\left(\Delta h_{t o t}\right)$ is calculated by:

$$
\Delta h_{t o t}=V_{s \phi}\left(t_{a}+t_{b}\right)+\Delta h_{g}
$$

The excess glide range can now be calculated, as excess height can be found from $h-\Delta h_{t o t}$.

$$
E_{g}=\gamma\left(h-\Delta h_{t o t}-152\right)
$$

where $152 \mathrm{~m}$ is the height required to fly final to land, which is $500 \mathrm{ft}$.

\section{REACHABILITY ANALYSIS EXAMPLE}

An example forced landing situation will now be considered. To show that this method for reachability analysis is applicable to a variety of aircraft with different sizes and velocities, an Airbus A320-400 will be utilised in this study and a Cessna 172 in Section VI. This is a fast low drag aircraft which has a very large turn radius, this would have made the previous method fail [8]. While the A320 is not an UAS and as it is also not single engined, the incident that made an American Airlines A320 perform a forced landing into the Hudson River shows that total engine failure can happen to any aircraft. All the parameters needed for the glide calculations are contained in Table 1, and many of these parameters have been obtained from [20].

The A320 had just taken off from a runway heading of $020^{\circ}, 12.2 \mathrm{~km}$ north of the runway climbing past $1200 \mathrm{~m}(\mathrm{agl})$, when it experienced a double engine failure. The only available landing site was the runway that it took off from. It is 
assumed that the engine failure occurred at $V_{i o}$. The calculations shown in this paper will be used to find out if the runway is reachable for this example. All the aircraft and runway initial conditions are shown below:

$$
\begin{array}{lll}
\psi_{0}=20^{\circ} & \Gamma=125^{\circ} & F_{x}=-1227 m \\
F_{y}=-9000 & x_{0}=0 & y_{0}=0 \\
V_{w}=30 \mathrm{~m} / \mathrm{s} & \phi=45^{\circ} & h_{\text {init }}=1200 \mathrm{~m}(\mathrm{agl})
\end{array}
$$

As the aircraft was travelling at a high airspeed of $112 \mathrm{~m} / \mathrm{s}$, it will be unaffected by a light wind, therefore an a unrealistically high wind speed of $30 \mathrm{~m} / \mathrm{s}$ from $330^{\circ}$ is used to give a $\beta$ of 0.268 to highlight the differences between wind and nil wind conditions.

If the $\psi_{w}$ is set to zero, the four possible trajectories in nil wind can be found, as shown in Fig. 8. In this case these equations essentially represent Dubins paths. The height lost by the aircraft at the end of the second turn is shown in Table 2. The trajectory chosen is the one which loses least height, which in this case is the path where the initial turn is clockwise $\left(\delta_{1}\right.$ is +1$)$ and the second turn is anti-clockwise $\left(\delta_{2}\right.$ is -1$)$. In the case of nil wind the chosen trajectory will be the path which subtends the smallest angle across both turns as it is the shortest. However this may not be the case where wind is present.

In the case where the wind blows at $30 \mathrm{~m} / \mathrm{s}$ from $330^{\circ}$, the four possible trajectories are also shown in Fig. 8 . The height

TABLE 1: Parameters for the A320-400.

\begin{tabular}{ll}
\hline Parameter & Value \\
\hline Cdo (clean) & 0.022 \\
$\mathrm{~S}$ & $122.5 \mathrm{~m}^{2}$ \\
$A_{r}$ & 9.5 \\
$\mathrm{k}$ & 1.2992 \\
$\mathrm{~W}$ & $671108 \mathrm{~N}$ \\
$\mathrm{~A}$ & $2.460 \times 10^{-} 6$ \\
$\mathrm{~B}$ & 389.3 \\
$V_{i o}$ & $112 \mathrm{~m} / \mathrm{s}$ \\
$\gamma$ & 16.2 \\
$V_{\text {sio }} \quad \phi=45^{\circ}$ & $9.81 \mathrm{~m} / \mathrm{s}$ \\
\hline
\end{tabular}

TABLE 2: Total height loss over maneuvre for nil wind condition for all four possible trajectories.

\begin{tabular}{cclll}
\hline$\delta_{1}$ & $\delta_{2}$ & $\Delta h$ nil wind & $\Delta h$ in wind & Travel Distance in wind \\
\hline-1 & 1 & $1712 \mathrm{~m}$ & $1526 \mathrm{~m}$ & $19.0 \mathrm{~km}$ \\
1 & 1 & $1580 \mathrm{~m}$ & $2182 \mathrm{~m}$ & $27.3 \mathrm{~km}$ \\
1 & -1 & $1030 \mathrm{~m}$ & $1034 \mathrm{~m}$ & $14.2 \mathrm{~km}$ \\
-1 & -1 & $1047 \mathrm{~m}$ & $964 \mathrm{~m}$ & $12.9 \mathrm{~km}$ \\
\hline
\end{tabular}




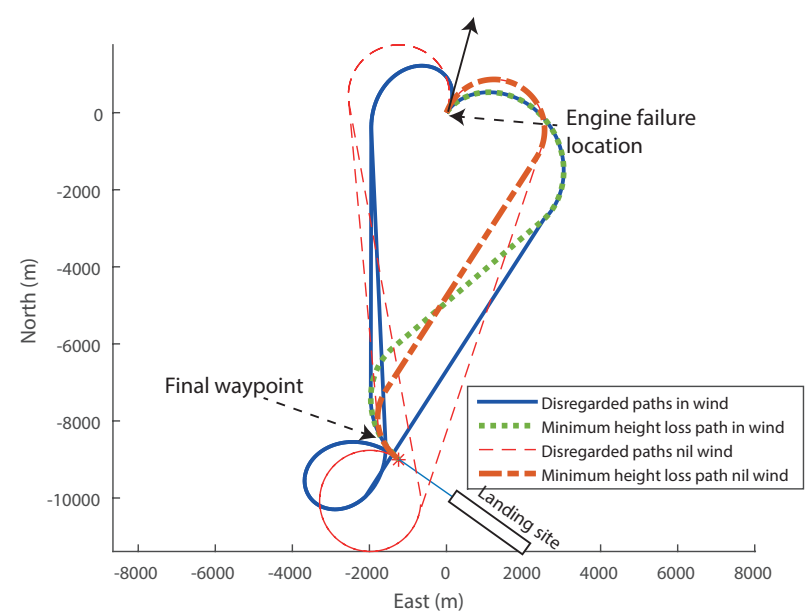

Fig. 8: Four possible maneuvres for the gliding aircraft to the runway in nil wind and $150^{\circ} 30 \mathrm{~m} / \mathrm{s}$ wind.

lost in the glide and the total path distances are shown in Table 2. In this case the optimum trajectory is where both turns are anti-clockwise i.e. $\delta_{1}=-1$ and $\delta_{2}=-1$, as it has the smallest height loss of $964 \mathrm{~m}$. It also has the shortest path length of $12.9 \mathrm{~km}$. However the shortest path in nil wind is not necessarily the shortest in wind. This is due to the possibility of higher ground speeds that could be enjoyed if a greater portion of that path has a higher tail wind component in the aircraft's direction of travel.

The best $\delta_{1}=-1$ and $\delta_{2}=-1$ trajectory is compared to the best no wind trajectory in Fig. 9. The optimal initial turn for both are different. This is because the initial turn with wind is into wind, as the wind direction blows the aircraft towards the landing site. This lowers the distance the aircraft has to travel in the level glide to $7071 \mathrm{~m}$ compared to $7271 \mathrm{~m}$ in nil wind. The A320 has a greater tail wind component in the level glide, where the aircraft's ground velocity is $138 \mathrm{~m} / \mathrm{s}$, whereas in nil wind it is travelling at its best glide speed of $V_{i o} 112 \mathrm{~m} / \mathrm{s}$. As there is an ever increasing tail wind component in the final turn, it loses significantly less height than the nil wind final turn at $138 \mathrm{~m}$, as opposed to $189 \mathrm{~m}$. The combination of these mean that the aircraft loses less height overall compared to not taking account of wind at all.

Shown in Fig. 10 are the height profiles of the aircraft over it's flight time. It shows the three distinct phases of the flight, with the turns having a steeper height loss as the sink rate is higher in a turn. The site is reachable if by the time it finishes the full manoeuvre, it is at or above 500ft $(152 \mathrm{~m})$. It can be seen that for both wind and nil wind both landing sites are reachable since the ends of the profiles are above $152 \mathrm{~m}$. As the wind is in a preferential direction, the trajectory in wind loses less height and thus has more excess height. The excess height in wind is $83.5 \mathrm{~m}$ and $17.2 \mathrm{~m}$, and the excess glide distance is $1380 \mathrm{~m}$ and $284 \mathrm{~m}$ respectively. If a forced landing was to be preformed in nil wind, the glide would be extremely marginal.

Paths are generated for the same initial and final conditions, but the wind is now from the South, which is much less favourable compared to the last direction. The best path is shown in Fig. 11: This is once again compared to the best nil 


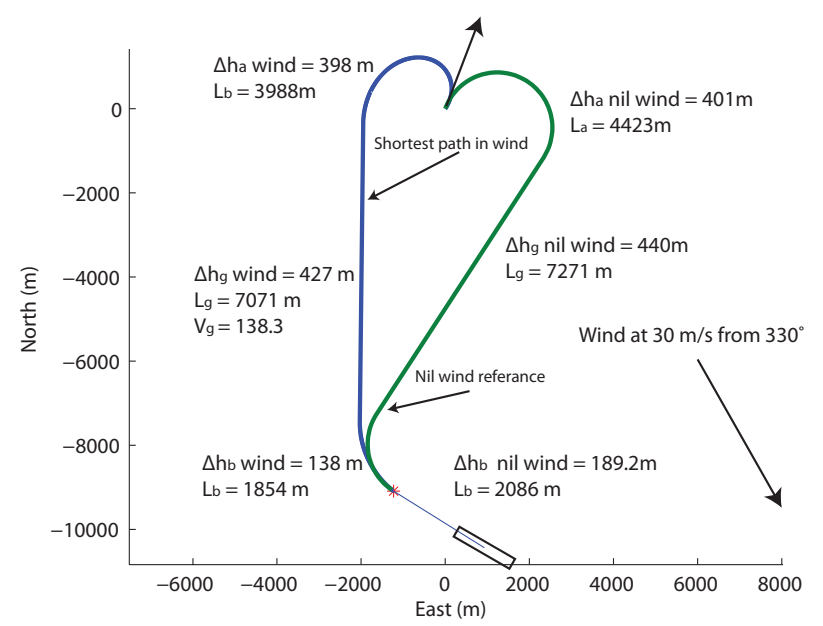

Fig. 9: Height profile of aircraft over shortest trajectory for wind from $330^{\circ} 30 \mathrm{~m} / \mathrm{s}$

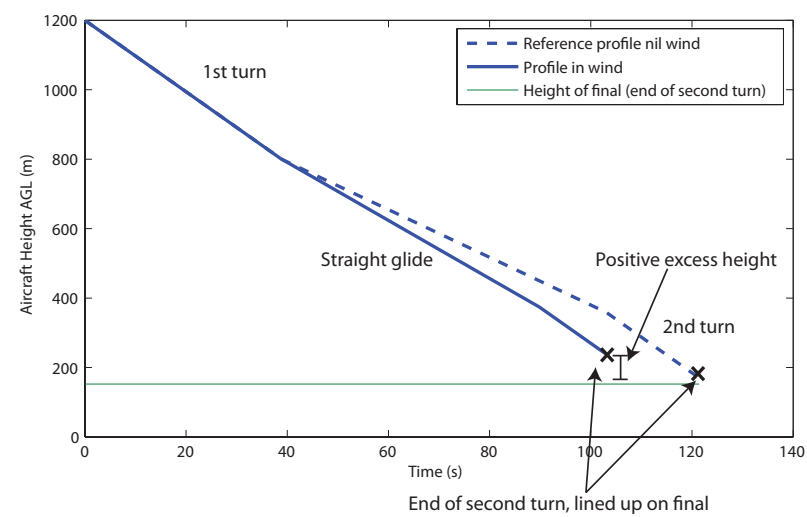

Fig. 10: Height profile of aircraft over best trajectory for wind from $330^{\circ} 30 \mathrm{~m} / \mathrm{s}$

wind path. In this case the best paths for wind and nil wind have the same initial turn direction. This is because the initial direction is at $020^{\circ}$ which is a turn into wind, and it minimises the distance in the first turn because the heading change is smaller.

It can be shown from the height profile in Fig. 12 that the aircraft has a negative excess height of $-47 \mathrm{~m}$, meaning that it does not have adequate height to reach this landing site due to the massively unfavourable wind conditions. This shows how important it is to take into account the wind.

\section{Vi. Monte Carlo simulation of Cessna 172}

To demonstrate that the algorithms presented in this paper make good height loss prediction despite the assumptions made, the method proposed is compared to actual height loss measured in the high fidelity X-Plane ${ }^{\circledR}$ simulation environment. The standard variable pitched propeller Cessna 172 model in X-Plane ${ }^{\circledR}$ will be used in these simulations. The aircraft dynamics model is based on blade element theory, meaning the aircraft has representative dynamics throughout the flight envelope. X-Plane ${ }^{\circledR}$ is used throughout industry for simulation and "XPlane has received FAA certification as a training simulator 


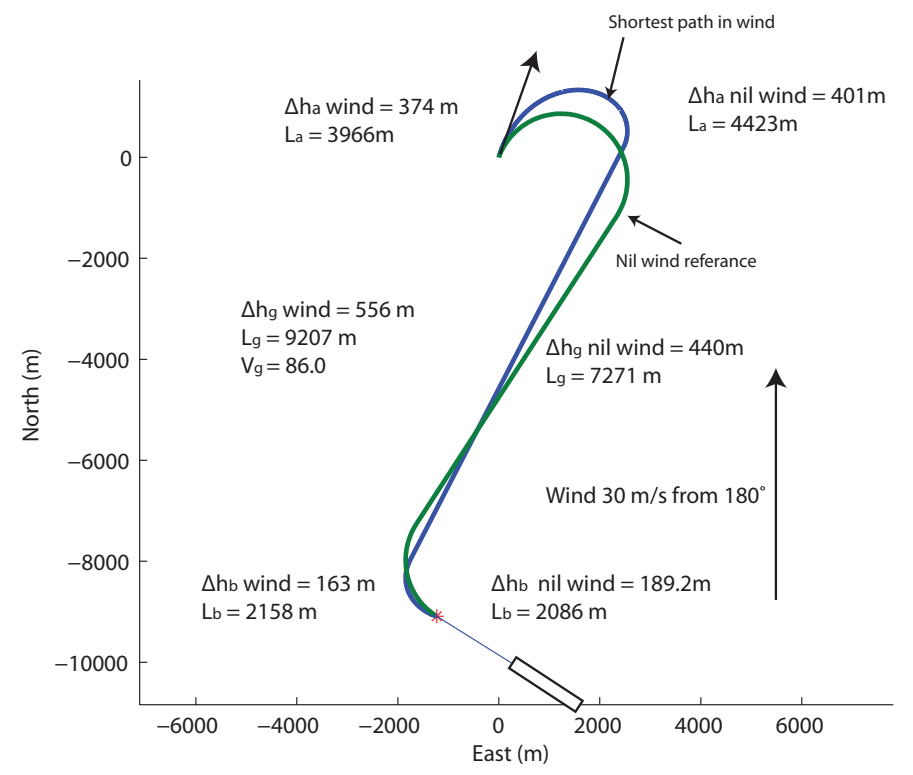

Fig. 11: Height profile of aircraft over best trajectory for wind from $180^{\circ} 30 \mathrm{~m} / \mathrm{s}$.

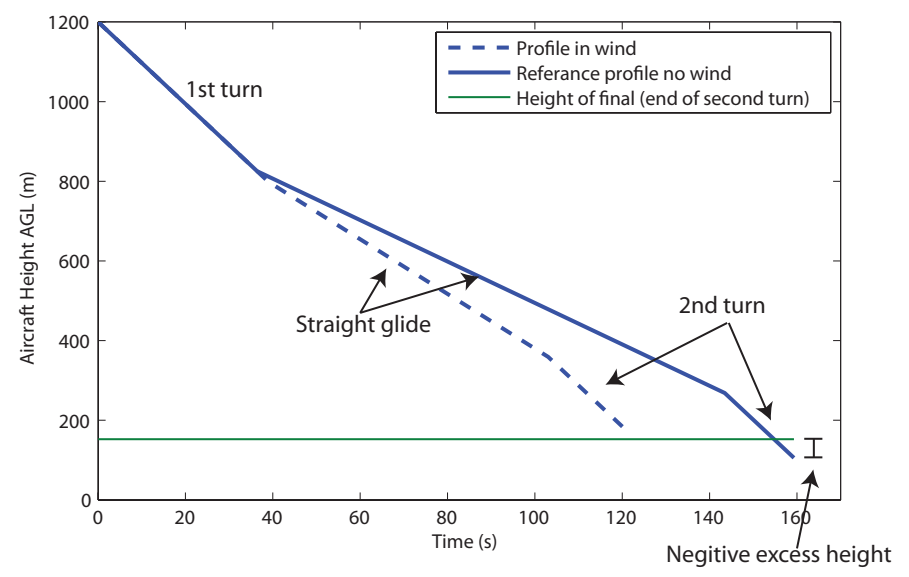

Fig. 12: Height profile of aircraft over best trajectory for wind from $180^{\circ} 30 \mathrm{~m} / \mathrm{s}$.

when used with certain hardware configurations because of its high fidelity simulation of flight model and visualization " [21]. The realism of this simulation means that X-Plane ${ }^{\circledR}$ is suitable for the following analysis.

A plugin was developed which gives access to all aircraft data like attitude, position etc. over a network port. The software can also receive control inputs like throttle, elevator etc. over the same network port. This has enabled the aircraft to be controlled externally by a separate computer. In Simulink a controller is setup to command the specific manoeuvres required, and is also used to log data. The relevant aircraft data from X-Plane ${ }^{\circledR}$ is fed into airspeed, roll angle and sideslip PID controllers. 


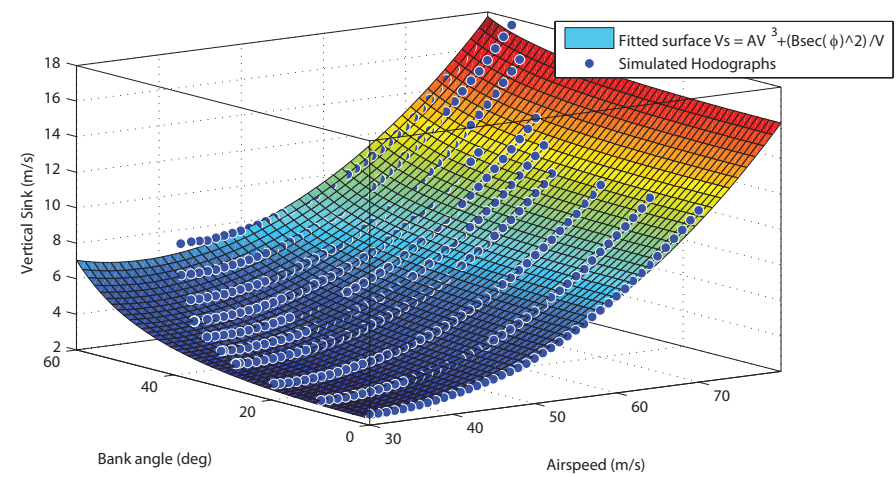

Fig. 13: Experimentally determined hodographs at a range of roll angles in X-Plane ${ }^{\circledR}$ compared to the fitted surface calculated from equations

\section{A. Experimental Determination of Glide performance}

The glide performance of the Cessna 172 needed to be determined experimentally as the aircraft's drag parameters were unknown. To show this method is applicable to a variety of aircraft with different sizes and velocities, an Airbus A320-400 and Cessna 172 will be utilized in the study. To achieve this the Cessna was be flown at a range of airspeeds to determine it's hodograph, which is it's vertical sink profile over a range of airspeeds.

These tests were repeated for a range of roll angles to determine the glide performance in the initial and final turns of the minimum height loss manoeuvre. This was also used to show the accuracy of Eq. 9, despite the assumptions made in it formulation.

The aircraft was initialised at $1500 \mathrm{~m}$, the throttle set to zero and the propeller feathered. The roll controller initially commanded a roll angle of zero, while the sideslip controller used the rudder to keep the sideslip of the aircraft at zero, which made any turns co-ordinated. The speed controller used the elevator to control the aircraft's airspeed to the commanded speed. The aircraft glided from the initial height of $1500 \mathrm{~m}$ to $1200 \mathrm{~m}$, giving its airspeed time to settle, and at this point the aircraft's vertical speed was recorded. The aircraft was reset to $1500 \mathrm{~m}$ and the demanded speed was decreased by $0.5 \mathrm{~m} / \mathrm{s}$.

The aircraft was put into a coordinated turn for a range of roll angles and a hodograph was recorded for each roll angle. The roll angles were $0^{\circ}, 10^{\circ}, 20^{\circ}, 30^{\circ}, 35^{\circ}, 40^{\circ}, 45^{\circ}, 50^{\circ}, 55^{\circ}$ and $60^{\circ}$. The turn was initialised by commanding the relevant roll angle for that run. The sideslip controller coordinated the turn. The hodographs for each run were fitted to the surface $V_{s}=f(V, \phi)$, where $f(V, \phi)$ is Eq. (9) This fitted surface is shown in Fig. 13.

The fit captured the shape of the experimentally determined hodographs well, with an R-Squared goodness of fit of 0.9936. The maximum error was around $-0.8 \mathrm{~m} / \mathrm{s}$ at high airspeeds and at a roll angle of $60^{\circ}$. However, these parts of the hodographs are mostly irrelevant, as the aircraft will be flying at $V_{i o}$. The error in vertical sink at $V_{i o}$ at $45^{\circ}$ roll angle was only 0.304 $\mathrm{m} / \mathrm{s}$.

The main glide performance figures obtained experimentally for the C-172 at a mass of 950kg are shown in Table 3. 
Some of these will be used in the next section, in order to perform height loss predictions.

TABLE 3: Main glide performance figures for the C-172

\begin{tabular}{ll}
\hline Parameter & Value \\
\hline$V_{\text {io }}$ & $37 \mathrm{~m} / \mathrm{s}$ \\
$V_{\text {sio }}$ & $2.825 \mathrm{~m} / \mathrm{s}$ \\
$\gamma_{\text {max }}$ & 13.09 \\
$V_{\text {mins }}$ & $31 \mathrm{~m} / \mathrm{s}$ \\
$V_{\text {smin }}$ & $2.603 \mathrm{~m} / \mathrm{s}$ \\
$\gamma_{\text {ms }}$ & 11.9 \\
$V_{\text {sio }}$ at $45^{\circ}$ roll & $4.348 \mathrm{~m} / \mathrm{s}$ \\
$\gamma$ at $45^{\circ}$ roll & 7.1297 \\
\hline
\end{tabular}

\section{B. Minimum height loss path simulation}

The minimum height loss paths were flown in the X-Plane ${ }^{\circledR}$ flight simulator as accurately as possible, the height loss and distance travelled across these paths measured and compared to predictions. X-Plane ${ }^{\circledR}$ were set up to fly these paths randomly in a simulated forced landing in order to conduct the Monte Carlo analysis.

X-Plane ${ }^{\circledR}$ was controlled in a similar way as in the previous section, but the demanded roll angle was commanded by a heading hold controller. The heading was set by Simulink's state flow tool, which commands an initial heading and an airspeed of $V_{i o}(37 \mathrm{~m} / \mathrm{s})$ at zero throttle, until it has settled on that heading and speed, following which the minimum height loss path was flown. This was done by commanding a new heading which initiates the initial turn, and then once on that heading, the aircraft conducted a straight-line glide for a set distance until the second turn, where the heading of the simulated runway was given as the heading command. The roll controller is aggressive, i.e a good deal of the turn is at maximum roll rate, in order to make it as efficient and as circular as possible. The headings and time in the glide can be entered manually or set randomly.

The start point of the initial turn (engine failure point) will be at $x_{0}=0, y_{0}=0 . \psi_{0}$ is the initial heading command of the aircraft, the straight line glide will be at a heading of $\psi_{a}$, while $\Gamma$ is the demanded heading for the second turn. $F_{x}, F_{y}$ are defined as the final points at the end of the second turn in metres. X-Plane ${ }^{\circledR}$ outputs the aircraft's location in either latitude and longitude or local cartesian coordinates in metres.

To make a comparison between X-Plane ${ }^{\circledR}$ and predictions, an example path will be shown with wind to demonstrate this method, and then a number of Monte Carlo simulations will be run for a range of turns, glide distances and wind conditions.

1) Simulation minimum height loss path examples: A strong wind of $20 \mathrm{kt}(10.28 \mathrm{~m} / \mathrm{s})$ from the east was chosen $(\beta=$ 0.277), which, when compared to $V_{i o}$, is high. The aircraft's initial random heading is $236^{\circ}$, and it then turns on to $59^{\circ}$ for its level glide and turns on to $\Gamma$ at $180^{\circ}$. Out of the four possible paths, the one that minimises height loss is an anti-clockwise 


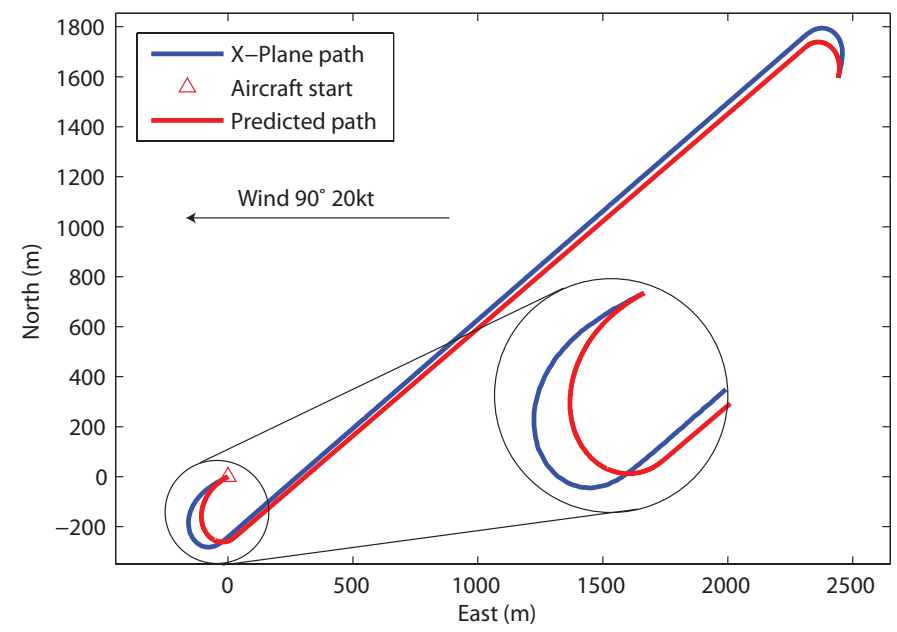

Fig. 14: Predicted compared to the simulated altitude profile for the $90^{\circ} 20 \mathrm{kt}$ wind example.

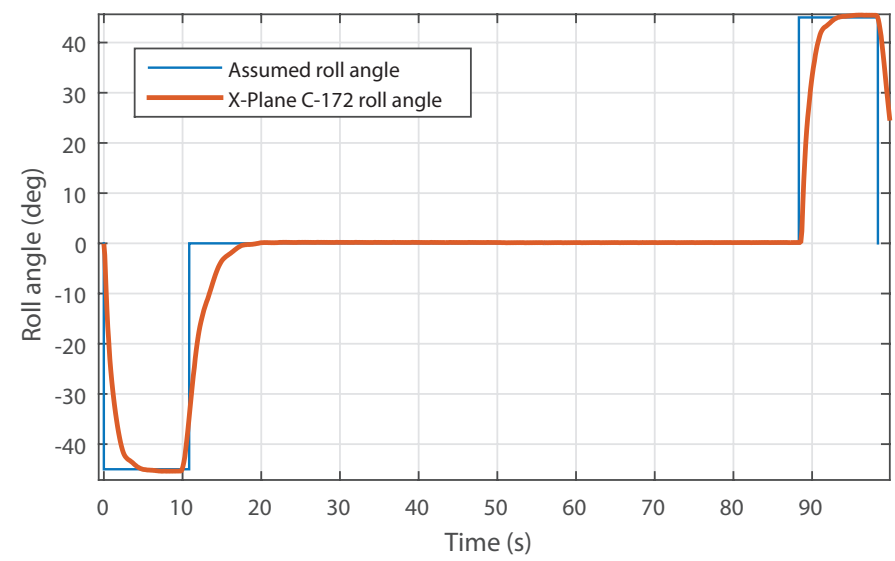

Fig. 15: Comparison of the assumed and simulated roll angles of the C-172 across the glide path.

turn followed by a clockwise turn. The path flown by the Cessna 172 is compared to the predicted path in Fig. 14. The paths are very similar. There is a blown up section of the final turn in the figure, which shows that in the steady wind of the X-Plane ${ }^{\circledR}$ simulation the turn shape is trochoidal - as predicted. The total path distance error is $115 \mathrm{~m}$, most of which comes from the initial turn. The assumed roll angle compared to the actual roll angle of the aircraft is shown in Fig. 15 which shows that the aircraft takes about 4 seconds to roll to the demanded angle. This lower yaw rate in the initial seconds after the engine failure explains the path distance error.

The height loss error is $20.9 \mathrm{~m}$, which is an error of $5 \%$ of the total height lost. The altitude profile of this glide can be seen in Fig. 16, they match closely in shape however, as shown in Table 4 as the first and final turn are slightly longer than predicted, and more height is lost over those phases, causing the majority of the error.

2) Monte Carlo simulations: So far, only a single example has been shown, and so in order to demonstrate how the height loss prediction calculation copes with a random range of headings and glide distances, with or without wind, a Monte Carlo simulation was run. 


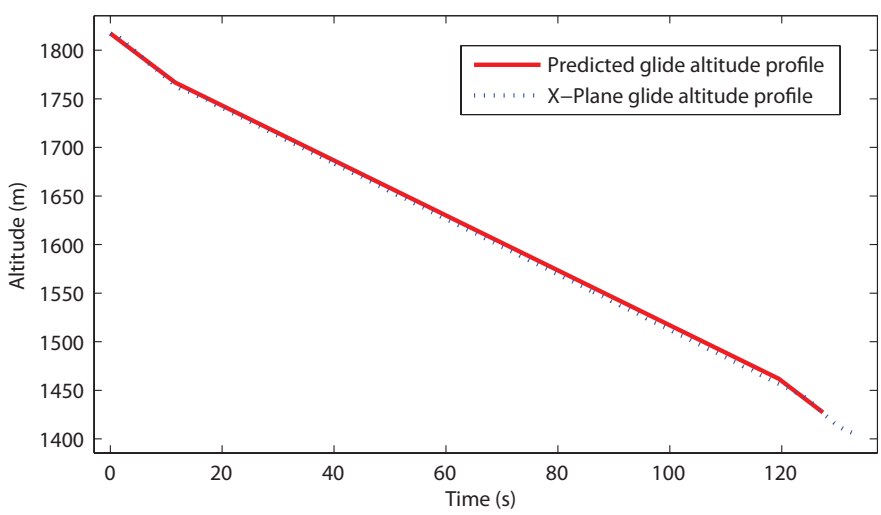

Fig. 16: Comparison of the predicted and simulated minimum height loss path for the $90^{\circ} 20 \mathrm{kt}$ wind example

The paths to fly were randomised for parameters $\psi_{0}, \Gamma$ randomised uniformly between $0^{\circ}-360^{\circ}$ and $L_{g}$ randomised uniformly between $2000 \mathrm{~m}$ to $20000 \mathrm{~m}$.

First of all, the simulation was run 95 times for nil wind conditions, which is to isolate the errors were not caused by the introduction of wind. A histogram of the height loss error between the predicted minimum height loss path and the actual height lost in the X-Plane ${ }^{\circledR}$ simulation is shown in Fig. 17a. The mean height loss error was $11.9 \mathrm{~m}$ with a standard deviation of $5.2 \mathrm{~m}$. This is a very small error which is negligible compared to the accuracy of barometric altitude, GPS altitude, and Digital Elevation Maps (DEM). Where barometric altimeters are certified to $15 \mathrm{~m}$ [22], GPS altitude is only accurate to $8.5 \mathrm{~m}$ [?], and the inaccuracies in DEM can vary greatly dependant on the source, however if Shuttle Radar Topography Mission DEM data was used for determining the landing site altitude, an accuracy of 15.27 RMSE was calculated in [23]. The error is constantly a positive error, meaning that the prediction consistently under-predicts height loss.

The distribution of the error in total path distance is shown in Fig. 17b. It has a mean of $100.5 \mathrm{~m}$ and a standard deviation of $51.8 \mathrm{~m}$, which means that the prediction is consistently under-predicting the path length.

TABLE 4: Comparison between predicted X-Plane ${ }^{\circledR}$ height loss and path distance for the $90^{\circ} 20 \mathrm{kt}$ wind example

\begin{tabular}{lll}
\hline Parameter & Predicted & Actual \\
\hline$\Delta h_{\text {tot }}$ & $390 \mathrm{~m}$ & $411 \mathrm{~m}$ \\
$\Delta h_{g}$ & $305 \mathrm{~m}$ & $312 \mathrm{~m}$ \\
$\Delta h_{a}$ & $50.6 \mathrm{~m}$ & $55.7 \mathrm{~m}$ \\
$\Delta h_{b}$ & $34.6 \mathrm{~m}$ & $43.5 \mathrm{~m}$ \\
$\Delta h_{e r r}$ & $20.9 \mathrm{~m}$ & \\
\hline$L$ & $3752 \mathrm{~m}$ & $3866 \mathrm{~m}$ \\
$L_{g}$ & $3016 \mathrm{~m}$ & $3085 \mathrm{~m}$ \\
$L_{a}$ & $401 \mathrm{~m}$ & $442 \mathrm{~m}$ \\
$L_{b}$ & $335 \mathrm{~m}$ & $340 \mathrm{~m}$ \\
$L_{e r r}$ & $115 \mathrm{~m}$ & \\
\hline
\end{tabular}




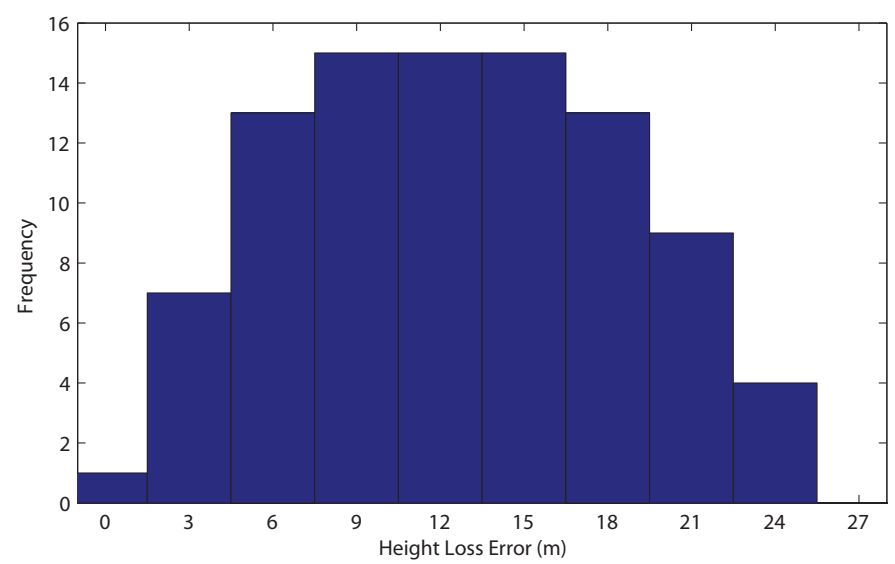

(a) Distribution of height loss error between the Monte Carlo simulation on X-Plane ${ }^{\circledR}$ and as predicted in nil wind.

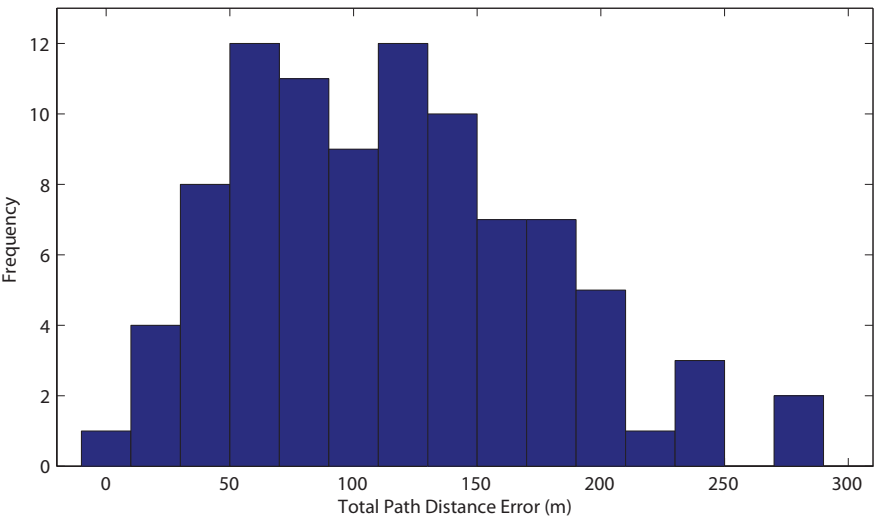

(b) Distribution of the total path length error between the Monte Carlo simulation on X-Plane ${ }^{\circledR}$ and as predicted in nil wind.

Fig. 17: Nil wind Monte Carlo simulation distributions.

As glide performance is accurately known, most of the errors in height loss come from disparities in the path length. To show that this correlation holds over a much larger sample size, the total path length error is plotted against the height loss error for each simulation run, as shown in Fig. 18a with a very strong positive correlation. If glide performance was not known accurately there would be a positive correlation between height loss error and $L_{g}$. However, no correlation is shown in Fig. 18b, meaning that in this case glide performance is known very accurately. This illustrates the importance of accurate glide performance information.

It is evident that the distance error was mostly caused by the instantaneous roll angle assumption. This can be further shown by a larger total heading change of the aircraft over the whole path, $\Delta \psi$ will lead to a greater total path length error. To show this correlation, total heading change over both turns is plotted against the total path distance error, as highlighted in Fig. 18c. Once again, this shows a positive correlation.

In the previous Monte Carlo simulation, the wind was steady and uniform. In order to see how the predictions would handle wind gusts, a Monte Carlo simulation with 103 runs was performed at a wind speed of 10kt from the north with 1 $\mathrm{m} / \mathrm{s}$ random wind gusts. X-Plane ${ }^{\circledR}$ makes this quite easy, as it has a weather option. The gusts are modelled as normally distributed, with $0 \mathrm{~m} / \mathrm{s}$ mean and standard deviation of $1 \mathrm{~m} / \mathrm{s}$.

From the height loss distribution in Fig. 19a, it seems that there is no significant impact on the accuracy of the prediction. The height loss error mean is $11.47 \mathrm{~m}$ with a standard deviation of $8.65 \mathrm{~m}$. This is comparable to stronger uniform steady wind Monte Carlo simulation presented earlier in this section.

The total path length error distribution is shown in Fig. $19 \mathrm{~b}$ with a mean of $106.5 \mathrm{~m}$ and a standard deviation of $66.74 \mathrm{~m}$. This also does not seem to have been unduly affected by the wind gust. This would make sense as the gusts have a mean of zero, therefore over time the error associated with integration of any vertical speeds error will be zero. 

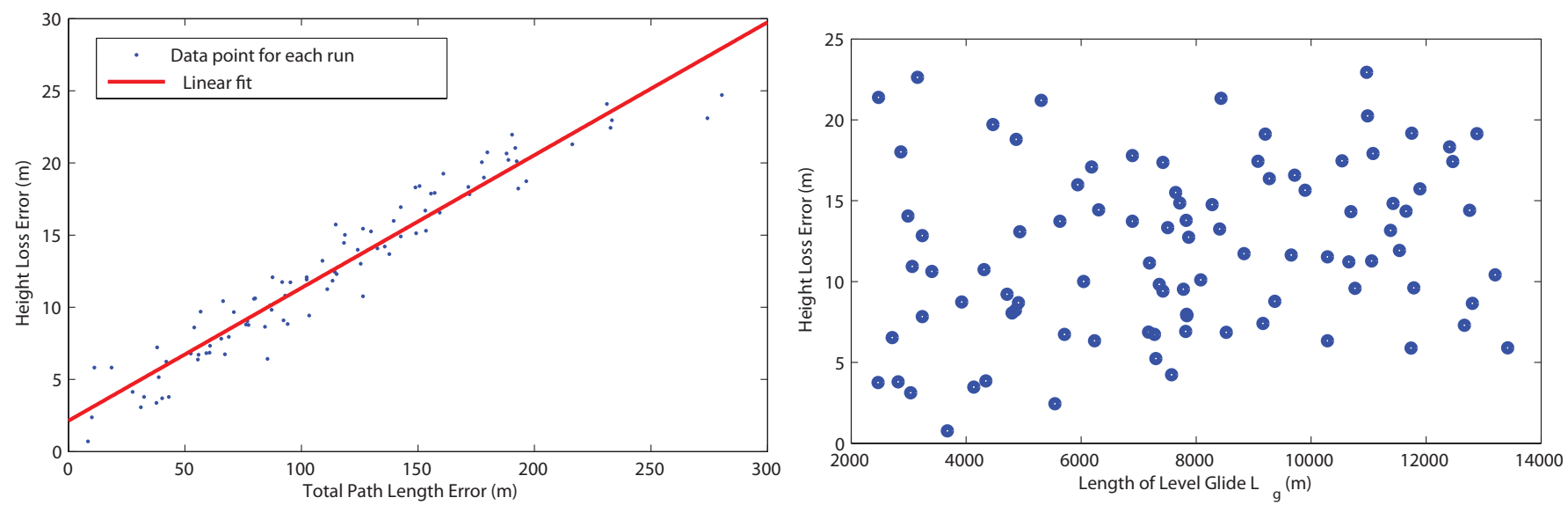

(a) Total path length error plotted against the height loss error for (b) Height loss error plotted against $L_{g}$ for each simulation run in each simulation run in nil wind. nil wind.

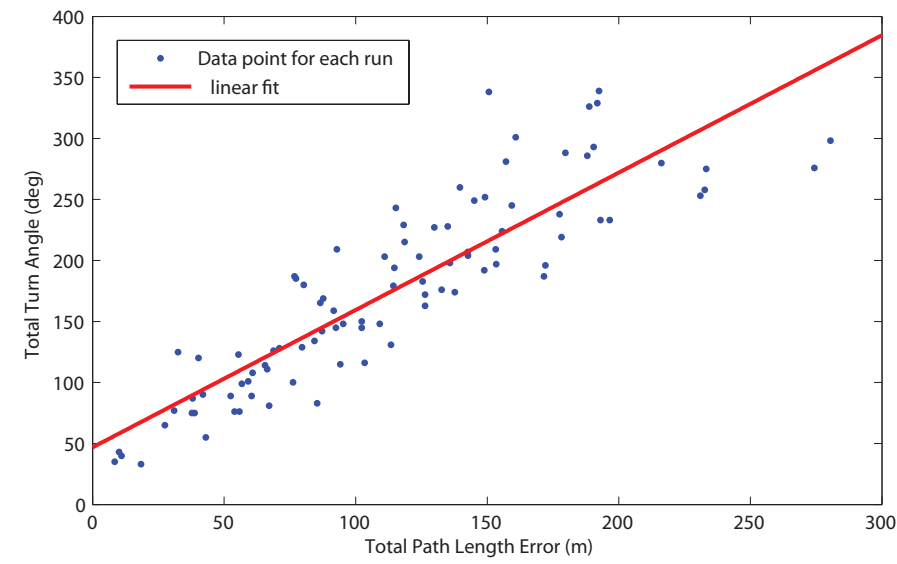

(c) Predicted compared to the simulated minimum height loss path nil wind.

Fig. 18: Graphs showing correlations between path length error and height error, and turn angle and path length error.

\section{CONCLUSION}

From a small number of initial conditions and aircraft parameters, reachability analysis for UAS has been developed as part of a contingency management system in a forced landing situation in the presence of a steady uniform wind. This is achieved by calculating the minimum height loss path of the gliding aircraft under wind conditions. The reachability analysis gives a measure of how reachable a landing site is by taking into account all the factors that affect an aircraft gliding and manoeuvring in performing a forced landing. This reachability measure is then used as a tool to aid landing site decision making.

By using a Cessna C-172 in an X-Plane ${ }^{\circledR}$ simulation environment, it has been shown that the experimentally determined hodographs match the predictions from the equations for both a straight-line glide and turning flight. This confirms that these simplified equations are adequate enough to model the glide performance for use in the height loss predictions. It has 


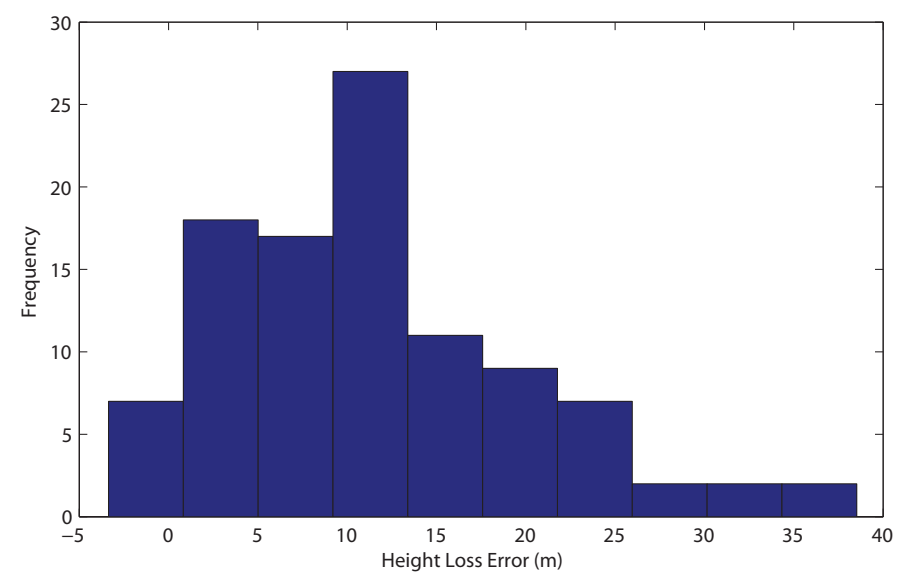

(a) Distribution of height loss error.

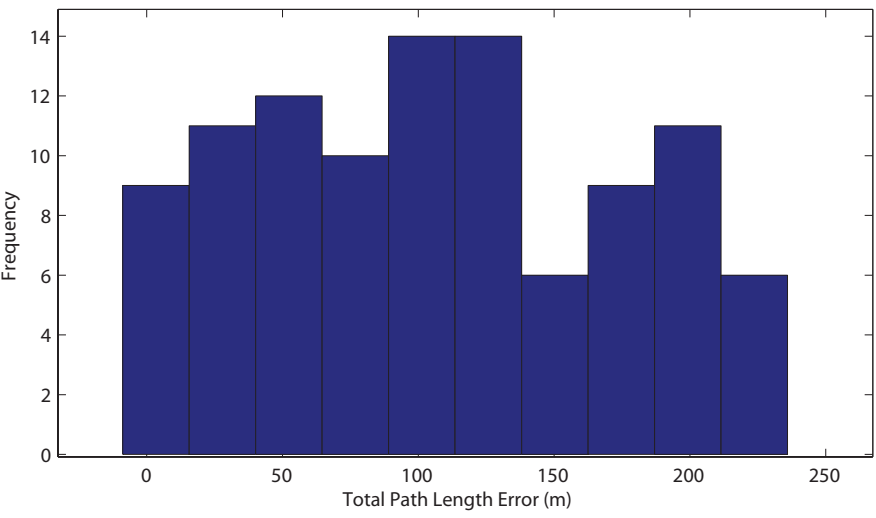

(b) Distribution of total path length error.

Fig. 19: Wind with gusts Monte Carlo simulation distributions between the Monte Carlo simulation in X-Plane ${ }^{\circledR}$ and as predicted, in $00^{\circ} 10 \mathrm{kt}$ wind with $1 \mathrm{~m} / \mathrm{s}$ random gusts.

also been shown that the proposed algorithm is fast enough to be run online.

The minimum height loss paths were investigated by comparing the predicted paths and height loss with simulated values from X-Plane ${ }^{\circledR}$ for the same C-172. Two examples showed the prediction methods perform well in both strong steady and gusty wind conditions. Monte Carlo simulations showed that the average height loss errors were quite small compared to the total height loss across the glide. Most of the total path length errors were the result of turns. This is because it is assumed that the aircraft instantaneously attains its desired roll angle. As this is not the case, the turns tended to be wider in the simulation, thereby increasing path length.

Finally, the proposed reachability analysis could also form part of the decision making tool for human pilots when a forced landing has to be performed.

\section{ACKNOWLEDGMENT}

This work was supported by the U.K. Engineering and Physical Sciences Research Council (EPSRC), Autonomous and Intelligent Systems programme under the grant number EP/J011525/1 with BAE Systems as the leading industrial partner.

\section{REFERENCES}

[1] NTSB, "Review of U.S. civil aviation accidents," National Transportation Safety Board, Tech. Rep. NTSB/ARA-12/01, 2010.

[2] T. H. Cox, C. J. Nagy, M. A. Skoog, I. A. Somers, and R. Warner, "A report overview of the civil uav capability assessment," National Aeronautics and Space Administration, 2005.

[3] D. Fitzgerald, R. Walker, and D. Campbell, "A vision based forced landing site selection system for an autonomous uav," in Intelligent Sensors, Sensor Networks and Information Processing Conference, 2005. Proceedings of the 2005 International Conference on, dec. 2005 , pp. 397 - 402. doi:10.1109/ISSNIP.2005.1595612 
[4] D. L. Fitzgerald, R. A. Walker, and D. A. Campbell, "A computationally intellgent framework for uav forced landings," in IASTED Computational Intelligence Conference. Calgary, Canada: ACTA Press, 2005.

[5] L. Mejias and D. Fitzgerald, "A multi-layered approach for site detection in uas emergency landing scenarios using geometry-based image segmentation," in Unmanned Aircraft Systems (ICUAS), 2013 International Conference on. IEEE, 2013, pp. 366-372. doi: 10.1109/ICUAS.2013.6564710

[6] P. C. Eng, "Path planning, guidance and control for a uav forced landing," Ph.D. dissertation, Queensland University of Technology, 2011.

[7] L. Mejias and P. C. Eng, "Experimental validation of an unpowered unmanned aerial system : application to forced landing scenarios," in 2012 International Conference on Unmanned Aircraft Systems (ICUAS'12), Sheraton Philadelphia University City Hotel, Philadelphia, PA, June 2012.

[8] M. Coombes, W.-H. Chen, and P. Render, "Reachability analysis of landing sites for forced landing of a uas," Journal of Intelligent \& Robotic Systems, vol. 73, no. 1-4, pp. 635-653, 2014. doi: 10.1007/s10846-013-9920-9

[9] D. F. Rogers, "Possible 'impossible' turn," Journal of Aircraft, vol. 32, no. 2, pp. 392-397, 1995.

[10] K. Brinkman and H. Visser, "Optimal turn-back manoeuvre after engine failure in a single-engine aircraft during climb-out," Proceedings of the Institution of Mechanical Engineers, Part G: Journal of Aerospace Engineering, vol. 221, no. 1, pp. 17-27, 2007. doi: 10.1243/09544100JAERO116

[11] D. C. Hyde, "Minimum-altitude-loss gliding turns with terminal constraints(return to runway after engine failure)," in 2005 AIAA Atmospheric Flight Mechanics Conference and Exhibit, 2005, pp. 1-17.

[12] M. J. S. Ella M. Atkins, Igor Alonso Portillo, "Emergency flight planning applied to total loss of thurst," Journal of Aircraft, vol. 43, pp. 1205-1216, 2006. doi: $10.2514 / 1.18816$

[13] D. Robson and D. Pooley, Flying Training, 8th ed., A. P. Publishing, Ed. Air Pilot Publishing, 2008.

[14] L. E. Dubins, "On curves of minimal length with a constraint on average curvature, and with prescribed initial and terminal positions and tangents," American Journal of mathematics, pp. 497-516, 1957. DOI: 10.2307/2372560

[15] D. Pooley, JAR Flying Training, H. H. Peter Godwin, Daljeet Gill, Ed. Pooley Aviation Ltd., 2003.

[16] L. Techy and C. A. Woolsey, "Minimum-time path planning for unmanned aerial vehicles in steady uniform winds," Journal of guidance, control, and dynamics, vol. 32, no. 6, pp. 1736-1746, 2009. DOI: 10.2514/1.44580

[17] M. Seleck, P. Váňa, M. Rollo, and T. Meiser, "Wind corrections in flight path planning," Int J Adv Robotic Sy, vol. 10, no. 248, 2013. DOI: $10.5772 / 56455$

[18] “The bisection algorithm," in Practical Analysis in One Variable, ser. Undergraduate Texts in Mathematics. Springer New York, 2002, pp. 165-177. [Online]. Available: http://dx.doi.org/10.1007/0-387-22644-3_14

[19] M. Abramowitz and I. A. Stegun, Handbook of mathematical functions: with formulas, graphs, and mathematical tables. Courier Dover Publications, 2012.

[20] M. L. V. Francois DE GERNON and A. RIGALDO, "Performance and design of the airbus a320," Department of Aerospace and Vehicle Engineering Royal Institute of Technology, Tech. Rep., December 2009.

[21] J. Craighead, R. Murphy, J. Burke, and B. Goldiez, "A survey of commercial open source unmanned vehicle simulators," in Robotics and Automation, 2007 IEEE International Conference on, april 2007, pp. 852 -857. DOI: 10.1109/ROBOT.2007.363092

[22] R. Pooley, Radio Navigation and Instrument Flying, 7th ed., ser. The Air Pilots Manual, J. S. Dorothy Pooley, Ed. Air Pilot Publishing, 2012, vol. Volume 5.

[23] M. L. Tighe and D. Chamberlain, "Accuracy comparison of the srtm, aster, ned, nextmap $₫$ usa digital terrain model over several usa study sites," in Proceedings of the ASPRS/MAPPS 2009 Fall Conference, San Antonio, TX, 2009. 